\title{
Additional Benefit of Chinese Medicine Formulae Including Dioscoreae rhizome (Shanyao) for Diabetes Mellitus: Current State of Evidence
}

Lu Sun ${ }^{1,2,3,4 \dagger}$, Yuan Ming Di ${ }^{5 \dagger}$, Chuanjian Lu $u^{1,3,4,5}$, Xinfeng Guo ${ }^{1,2,3,4}$, Xianyu Tang ${ }^{1,2,3,4}$, Anthony Lin Zhang ${ }^{5}$, Charlie Changli Xue ${ }^{1,3,4,5 *}$ and Guanjie Fan ${ }^{1,2,3,4 *}$

\section{OPEN ACCESS}

Edited by:

Wenquan Zou,

Case Western Reserve University, United States

Reviewed by: Aiping Lu,

Tongji University, China Guo-qing Zheng,

The Second Affiliated Hospital \& Yuying Children's Hospital of Wenzhou Medical University, China

*Correspondence:

Charlie Changli Xue charlie.xue@rmit.edu.au Guanjie Fan

13925119990@139.com

${ }^{\dagger}$ These authors have contributed equally to this work

Specialty section: This article was submitted to Clinical Diabetes, a section of the journal Frontiers in Endocrinology

Received: 18 April 2020 Accepted: 14 October 2020 Published: 10 November 2020

Citation:

Sun L, Di YM, LU C, Guo X, Tang X, Zhang AL, Xue CC and Fan G (2020) Additional Benefit of Chinese Medicine

Formulae Including Dioscoreae rhizome (Shanyao) for Diabetes Mellitus: Current State of Evidence.

Front. Endocrinol. 11:553288. doi: 10.3389/fendo.2020.553288

\begin{abstract}
${ }^{1}$ The Second Affiliated Hospital of Guangzhou University of Chinese Medicine, Guangzhou, China, ${ }^{2}$ The Second Clinical College of Guangzhou University of Chinese Medicine, Guangzhou, China, ${ }^{3}$ Guangdong Provincial Hospital of Chinese Medicine, Guangzhou, China, ${ }^{4}$ Guangdong Provincial Academy of Chinese Medical Sciences, Guangzhou, China, ${ }^{5}$ The China-Australia International Research Centre for Chinese Medicine, School of Health and Biomedical Sciences, RMIT University, Melbourne, VIC, Australia
\end{abstract}

Background: Chinese medicine has been used to treat diabetes symptoms for thousands of years. Dioscoreae rhizome or Shanyao is a Chinese medicinal herb that is routinely used in the treatment of diabetes mellitus (DM).

Objective: The purpose of this study is to evaluate the evidence of the added benefits and safety of herbal formulae containing Shanyao in clinical studies and the possible mechanisms of Shanyao in the prevention and treatment of DM in experimental studies.

Methods: We searched nine databases for randomized controlled trials (RCTs) that included Shanyao in the formulae in the treatment of type 2 DM. Furthermore, experimental studies on the prevention and treatment of DM by Shanyao in Englishand Chinese-language databases were identified.

Results: Fifty-three moderate quality RCTs with herbal formulae containing Shanyao were identified. Results from meta-analysis indicated that Shanyao alone or formulae containing Shanyao in addition to conventional treatments could benefit people with type 2 DM in lowering blood glucose, blood lipids and reducing insulin resistance. Moreover, adverse events were significantly lower in the CHM plus conventional group than those in the conventional group. Shanyao may exert the benefit through various mechanisms including inhibition of $\alpha$-glucosidase and DPP-IV activity, increase of endogenous GLP-1 and immune regulating activities.

Conclusion: Evidence from this review suggested that there appeared to be added clinical benefits associated with the use of Shanyao for DM, whether as a food supplement or as a CHM combined with hypoglycemic agents with a good safety profile.

Keywords: Dioscoreae rhizome, Shan yao, Chinese medicine, diabetes, systematic review, efficacy, safety, mechanism 


\section{INTRODUCTION}

Diabetes mellitus (DM) prevalence is growing at an alarming rate worldwide. According to the International Diabetes Federation, about 463 million adults worldwide suffer from DM (1). Among them, China is the country with the largest number of people suffering from DM.

Approximately $95 \%$ of the diabetic population is T2DM, followed by type 1 diabetes mellitus, gestational diabetes mellitus, and specific types of diabetes due to other causes (2). Patients with type 1 diabetes mellitus and gestational diabetes mellitus are mainly treated with insulin supplementation and replacement therapy. Specific etiology and pathogenesis of other types of DM vary, and the treatment plan is not unified. Therefore, this study focuses on patients with T2DM.

Epidemiological figures showed that the prevalence of type 2 diabetes mellitus (T2DM) in adults aged 18 and over in China is $10.9 \%$ and that of pre-diabetes is $35.7 \%$ (3). T2DM is a chronic progressive disease. Da Qing research, a registry cohort study with long term follow-up in China showed that $67.7 \%$ of prediabetic people will develop DM after six years without early intervention, but the risk of T2DM in the combined diet and exercise group can be reduced by $42 \%$ (4). UK Prospective Diabetes Study suggested that strict glycemic control can reduce the risk of diabetic complications (5). Early active management can be more beneficial for diabetic people, which coincides with the idea of "cure disease before disease onset" in traditional Chinese medicine (TCM).

Treating diabetes by conventional medicine includes oral and injectable hypoglycemic agents. Diabetic patients often need a combination of several drugs. Common concerns of doctors and diabetic patients include hypoglycemia, gastrointestinal discomfort, inconvenience of missing medicines and injectable medicines. The Chinese Diabetes Society (CDS) guidelines of 2017 presented information of TCM for diabetes and recommended the use of TCM (6). In China, it has been observed that doctors in the field of diabetes are also looking for diverse treatments including TCM (6).

In TCM, DM belongs to the category of disease called " $x i a o$ ke 消渴”. Disease characteristics include thirst, excessive drinking, polyuria, and weight loss. The pathogenesis of DM in TCM is qi and yin deficiency and excessive dryness and heat. Commonly used formulae by TCM practitioners include Liu wei di huang wan 六味地黄丸, Shen qi wan 肾气丸, and Ba wei wan八味丸 (7). Shanyao (Dioscoreae rhizome) is the root of Dioscorea opposita Thunb. (8); it is one of the main herbs in the above formulae. Shanyao has been widely used in the treatment of xiao ke消渴or diabetes since the ancient times. It is a popular herb and can also be consumed as a type of food. Active ingredients of Shanyao include polysaccharides, flavonoids, allantoin, choline, and dioscin (9-14).

This study will review evidence of Shanyao for DM from clinical research and experiment research results from Chineseand English-language databases and present the evidence on added benefits and safety of herbal formula containing Shanyao in clinical studies. Possible mechanisms of Shanyao in the prevention and treatment of DM in experimental studies are also investigated.

\section{METHODS}

\section{Systematic Review of Clinical Trials Search Strategy}

We searched English- and Chinese-language databases and followed the methods outlined in the Cochrane Handbook of Systematic Reviews (15). English-language databases included PubMed, ExcerptaMedica Database (Embase), Cumulative Index of Nursing and Allied Health Literature (CINAHL), Cochrane Central Register of Controlled Trials (CENTRAL), including the Cochrane Library, and Allied and Complementary Medicine Database (AMED); Chinese-language databases included China SinoMed, China National Knowledge Infrastructure (CNKI), Chongqing VIP (CQVIP), and Wanfang. Databases were searched from inception to March 2019. No restrictions were applied.

Search terms were grouped into three blocks: 1) intervention (formulae including Shanyao, dioscoreae rhizome); 2) clinical condition (including type 2 diabetes mellitus); and 3) trial design (including clinical trial, randomized controlled trial).

We also searched reference lists of previous systematic reviews and included studies. Clinical trial registries were also searched including the Australian New Zealand Clinical Trial Registry (ANZCTR), Chinese Clinical Trial Registry (ChiCTR), European Union Clinical Trials Register (EU-CTR) and USA National Institutes of Health register (ClinicalTrials.gov). When required, we contacted trial investigators by email or telephone to obtain data. If we didn't receive a response after four weeks, we marked the unknown information 'not available'.

Protocol of the review was registered with the PROSPERO international prospective register of systematic reviews (CRD 42019145668).

\section{Study Inclusion Criteria \\ Study design \\ Randomized controlled trials (RCTs) were eligible.}

\section{Participants}

Adults were diagnosed with T2DM using the following guidelines: 1999 World Health Organization (WHO) (16), Chinese Diabetes Society $(17,18)$, American Diabetes Association (2), or a description of diagnostic criteria including:

- Fasting blood glucose (FBG, defined as no caloric intake for at least $8 \mathrm{~h}) \geq 126 \mathrm{mg} / \mathrm{dl}(7.0 \mathrm{mmol} / \mathrm{L})$,

- Or 2 -h plasma glucose $(P G) \geq 200 \mathrm{mg} / \mathrm{dl}(11.1 \mathrm{mmol} / \mathrm{L})$ during an oral glucose tolerance test (OGTT). The test should be performed as described by the WHO, using a glucose load containing the equivalent of $75 \mathrm{~g}$ anhydrous glucose dissolved in water.

- Or $\mathrm{A} 1 \mathrm{C} \geq 6.5 \%$ ( $48 \mathrm{mmol} / \mathrm{mol})$. The test should be performed in a laboratory using a method that is National Glycohemoglobin Standardization Program certified and standardized to the Diabetes Control and Complications Trial assay. 
- Or in a patient with classic symptoms of hyperglycemia or hyperglycemic crisis, a random plasma glucose $\geq 200 \mathrm{mg} / \mathrm{dl}$ (11.1 mmol/L). In the absence of unequivocal hyperglycemia, results should be confirmed by repeat testing.

\section{Type of Interventions}

$\mathrm{CHM}$, other CM therapies (for example, Chinese medicine dietary therapy), and integrative medicine such as CHM plus hypoglycemic drugs; all the interventions should include Shanyao.

\section{Type of Controls}

Conventional therapies recommended in guidelines, including pharmacotherapy, diet therapy and lifestyle interventions.

\section{Outcomes}

The primary outcome measures were:

- Blood glucose tests (fasting blood glucose, post-prandial blood glucose, hemoglobin $\mathrm{a}_{1 \mathrm{c}}$ );

- Adverse events (AEs);

The secondary outcomes were:

- Blood lipid metabolism indicators (triglyceride, cholesterol, low-density lipoprotein, high-density lipoprotein);

- $\beta$-cell function indicators: fasting serum insulin (Fins) the unit of FINS is $\mathrm{pmol} / \mathrm{L}$ or $\mu \mathrm{u} / \mathrm{ml}$ will be included $(\mathrm{pmol} / \mathrm{L}=$ $\mu \mathrm{u} / \mathrm{ml} \times 6.965) ; \mathrm{HOMA}-\mathrm{IR}(\mathrm{IR})=\mathrm{FBG} \times \mathrm{FINS} / 22.5$; HOMAIS (IS) $=1 /($ Fins $\times$ FPG);

- Body Mass Index (BMI).

\section{Study Exclusion Criteria}

- Quasi-randomized controlled trials;

- Prediabetic state;

- Type 1 diabetes;

- Gestational diabetes;

- Other specific types of diabetes included in the American Diabetes Association and Chinese Diabetes Society:

- Genetic defects of beta-cells

- Genetic defects in insulin action

- Diseases of the exocrine pancreas

- Endocrinopathies

- Drug or chemical induced diabetes

- Infections

- Uncommon forms of immune-mediated diabetes

- Other genetic syndromes sometimes associated with diabetes

- Diabetic complications and comorbidities;

- Integrative medicine studies that used different therapies in the intervention group and control group;

- If the control group uses a form of Chinese medicine.

\section{Data Extraction and Management}

Search results were synthesized by removing duplicates, followed by screening of titles and abstracts by LS and YD. Full texts were obtained and screened by two reviewers (LS and YD). Eligible studies satisfying the inclusion criteria were extracted using EpiData software (EpiData Association, Odense, Denmark). LS and YD extracted the data from the included studies independently and double-checked the data to obtain information on authors, publication year, title, journal, participants' characteristics, sample size, methodological details, intervention details, treatment duration, outcome measures, and AEs.

\section{Assessment of Risk of Bias in Included Studies}

Risk of bias was assessed using the Cochrane Collaboration's procedures (15). RevMan software (Version 5.2.4, Copenhagen: The Nordic Cochrane Centre, The Cochrane Collaboration, 2012) was used for risk of bias analysis. Items of bias assessed included sequence generation, allocation concealment, blinding of participants, blinding of personnel, blinding of outcome assessment, incomplete outcome data, selective outcome reporting and other bias including baseline imbalance and funding. Egger's test was used to assess publication bias. Publication bias was assessed when the subgroup included more than 10 studies.

Risk of bias assessment was conducted by two independent reviewers (LS and YD) and disagreement was resolved by discussion or consultation with a third person (TZ).

\section{Data Analysis}

Continuous outcomes were presented as mean difference (MD) with 95\% confidence interval (CI) between two groups, whereas dichotomous data were presented as relative risk (RR) with $95 \%$ CI. Stata software (13.0) was used for data analysis. Considering heterogeneities among trials, all meta-analyses were performed with random effects model. Heterogeneities between studies was estimated by $I^{2}$. An $I^{2}$ score greater than $50 \%$ was considered to indicate substantial heterogeneity.

Predefined comparisons in the meta-analysis were as follows: (1) CHM plus hypoglycemic agents versus hypoglycemic agents,

(2) CHM plus lifestyle intervention versus lifestyle intervention,

(3) $\mathrm{CHM}$ diet therapy plus lifestyle intervention versus lifestyle intervention.

Subgroup analysis were performed where possible, including studies with low risk for sequence generation, FBG level at baseline $(6-\leq 8 \mathrm{mmol} / \mathrm{L}, 8-10 \mathrm{mmol} / \mathrm{L}, \geq 10 \mathrm{mmol} / \mathrm{L})$, patient age groups (18-40 years, 41-64 years, $>65$ years), BMI (normal < $24 \mathrm{~kg} / \mathrm{m}^{2}$, overweight $\geq 24-28 \mathrm{~kg} / \mathrm{m}^{2}$, obese $\geq 28 \mathrm{~kg} / \mathrm{m}^{2}$ ), disease duration ( $<5$ years, $\geq 5-10$ years, $\geq 10$ years), treatment duration ( $\leq 3$ months, 3-6 months, and $\geq 6$ months), comparator drugs class and CM syndrome differentiation (19-23).

The Grading of Recommendations Assessment, Development and Evaluation (GRADE) approach was used to assess the quality of evidence.

\section{Pharmacological Research Evidence of Shanyao for DM}

The constituent compounds were identified by searching herbal monographs, high quality reviews of CHM, pharmacopoeia of the People's Republic of China (24), and PubMed. To identify preclinical publications a literature search of PubMed and China 
National Knowledge Infrastructure was undertaken. The search strategy included the terms for Shanyao and its constituent compounds and T2DM. Relevant data were extracted, and a summary of the findings are reported here.

\section{RESULTS}

\section{Modern Literature Results Description of Included Studies Search Results}

Our search identified 44,958 articles in the included databases. Fifty-three (53) RCTs involving 4,905 participants were included in the systematic review (25-77). The screening process is shown in Figure 1.

\section{Characteristics of the Included Studies}

All studies were randomized, parallel-group, controlled trials conducted in China between 2002 and 2018. One study published was in English (44) and the rest in Chinese language. All studies included participants diagnosed in accordance with the 1999 WHO, Chinese Diabetes Society or American Diabetes Association diagnostic criteria for T2DM. In total, 4,905 participants were included in these RCTs; participants' age ranged from 45 to 74 years. Duration of T2DM ranged from 1 week to 20 years. Treatment duration ranged from 2 to 24 weeks. Only one study had a follow-up for 30 weeks (65). Characteristics of included studies are summarized in Table 1.

Fifty-three (53) RCTs assessing CHM as food or integrative medicine for T2DM were identified from the search. 51 studies assessed the combination of CHM with conventional medication (integrative medicine) (27-77). One study compared CHM to lifestyle intervention (26); one study compared Chinese medicine diet therapy to lifestyle intervention (25).
Ninety-eight herbs were used in the formulae, and the most commonly used herb used with Shanyao 山药 werehuangqi 黄芪 (42 studies), shengdihuang 生地黄 (30 studies), gegen 葛根 (28 studies), tianhuafen 天花粉 (28 studies), danshen 丹参 (26 studies), fuling 获苓 (23 studies), maimendong 麦门冬 (22 studies), shanzhuyu 山茱莫 (21 studies), and huanglian 黄连 (16 studies).

Comparators included pharmacologic therapy and lifestyle intervention. Pharmacologic therapy used in the included RCTs includes biguanides, sulfonylureas, thiazolidinediones, aglucosidase inhibitors, DPP-4 inhibitors, and insulins. Lifestyle management of T2DM includes diabetes self-management education and support, medical nutrition therapy, physical activity, and psychosocial care.

CM syndrome differentiation was described in thirty-four studies $(26,28,29,31-41,43,46,48-50,52,53,55,57,59-62,64$, $67,69,71,74,75,77)$. The most common syndromes described in the studies include $q i$ and yin deficiency 气阴两虚, yin deficiency and excessive heat 阴虚热盛, damp heat retention 湿热阻滞, spleen deficiency 脾虚, phlegm-dampness, and blood stasis 疢 湿血瘀.

\section{Risk of Bias in the Included Studies}

The results of the risk of bias judgements are presented in Figure 2 and Figure 3. All included 53 studies were randomized; 15 trials described the process of random sequence generation (25, 26, 31, 32, 34, 37, 38, 43, 44, 46, 62, 64, 65, 69, 73); two trials implemented allocation concealment $(25,37)$. One study was described as "single-blind" trial with no further details (37). Considering all included outcome measures were objective outcomes, blinding of assessors has low risk of influencing the outcome measures and was judged as low risk of bias in all included studies. Twelve trials did not report on all outcome measures described in the Methods section (37, 40, 44, 53, 56-58, $60,62,63,75,77)$, three trials reported on AEs which were not

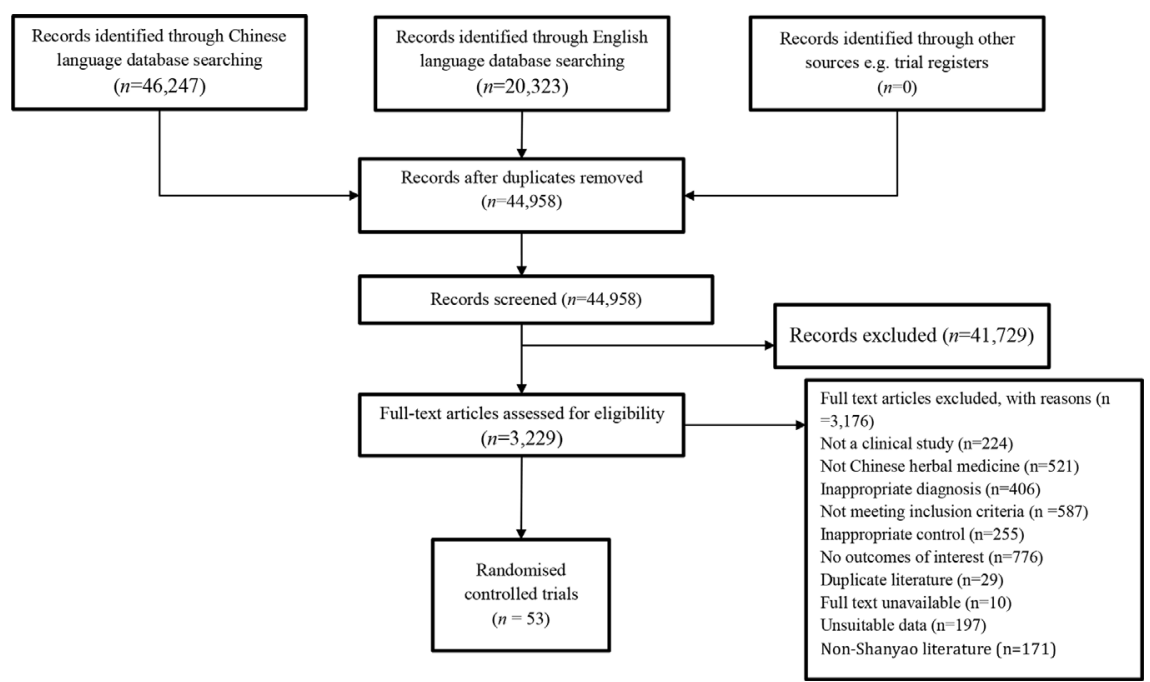

FIGURE 1 | Flow chart of study selection process. 
TABLE 1 | Basic characteristics of the included studies in modern literature.

\begin{tabular}{|c|c|c|c|c|c|c|c|c|c|c|c|c|c|c|c|c|c|c|c|c|}
\hline \multirow[t]{2}{*}{ No. } & \multirow[t]{2}{*}{ Study } & $\begin{array}{l}\text { Sample } \\
\text { size }\end{array}$ & $\begin{array}{l}\text { Sample } \\
\text { size }\end{array}$ & $\begin{array}{l}\text { Mean } \\
\text { age(y) }\end{array}$ & $\begin{array}{l}\text { Mean } \\
\text { age(y) }\end{array}$ & \multirow[t]{2}{*}{ Treatment } & \multirow[t]{2}{*}{ Control } & \multirow[t]{2}{*}{$\begin{array}{l}\text { Duration } \\
\text { (w) }\end{array}$} & \multirow[t]{2}{*}{ FBG } & \multirow[t]{2}{*}{$2 \mathrm{hPG}$} & \multirow[t]{2}{*}{$\mathrm{HbA}_{1 \mathrm{c}}$} & \multirow[t]{2}{*}{ TG } & \multirow[t]{2}{*}{ TC } & \multirow[t]{2}{*}{ LDL } & \multirow[t]{2}{*}{ HDL } & \multirow[t]{2}{*}{ BMI } & \multirow[t]{2}{*}{ FINS } & \multirow[t]{2}{*}{ IR } & \multirow[t]{2}{*}{ IS } & \multirow[t]{2}{*}{ AEs } \\
\hline & & (I) & (C) & (I) & (C) & & & & & & & & & & & & & & & \\
\hline 1 & Bai (42) & 40 & 30 & 48.27 & 49.23 & SMF\&Bigu\&Sulf & Bigu/Sulf & 4 & $\sqrt{ }$ & $\sqrt{ }$ & $x$ & $\sqrt{ }$ & $\sqrt{ }$ & $x$ & $x$ & $x$ & $x$ & $x$ & $x$ & $x$ \\
\hline 2 & Cao et al. (77) & 68 & 68 & 48 & 47 & SMF\&Bigu & Bigu & 8 & $\sqrt{ }$ & $\sqrt{ }$ & $\sqrt{ }$ & $\sqrt{ }$ & $\sqrt{ }$ & $x$ & $x$ & $x$ & $x$ & $x$ & $x$ & $\sqrt{ }$ \\
\hline 3 & Cao and Zou (76) & 54 & 54 & 56.7 & 54.5 & Baihurenshen Formula\&Sulf & Sulf & 8 & $\sqrt{ }$ & $\sqrt{ }$ & $x$ & $x$ & $x$ & $x$ & $x$ & $x$ & $\sqrt{ }$ & $\sqrt{ }$ & $\sqrt{ }$ & $\sqrt{ }$ \\
\hline 4 & Chen (30) & 24 & 26 & 60.33 & 61.35 & a-Glucosidase or Insulins & $\begin{array}{l}\text { a-Glucosidase/ } \\
\text { Insulins }\end{array}$ & 6 & $\sqrt{ }$ & $\sqrt{ }$ & $\sqrt{ }$ & $\sqrt{ }$ & $\sqrt{ }$ & $\sqrt{ }$ & $\sqrt{ }$ & $x$ & $x$ & $x$ & $x$ & $\sqrt{ }$ \\
\hline 5 & Cui and Lou (75) & 40 & 40 & 56.75 & 52.57 & SMF\&Bigu\&Sulf & Bigu\&Sulf & 8 & $\sqrt{ }$ & $\sqrt{ }$ & $\sqrt{ }$ & $x$ & $x$ & $x$ & $x$ & $x$ & $x$ & $x$ & $x$ & $x$ \\
\hline 6 & Fan et al. (74) & 40 & 40 & $42-74$ & $40-73$ & Shenqijiangtang Granule\&Bigu & Bigu & 12 & $\sqrt{ }$ & $\sqrt{ }$ & $\sqrt{ }$ & $x$ & $x$ & $x$ & $x$ & $x$ & $x$ & $x$ & $x$ & $x$ \\
\hline 7 & Gao et al. (73) & 90 & 90 & 58.9 & 57.6 & Shenqijiangtang Capsule\&Bigu & Bigu & 12 & $\sqrt{ }$ & $\sqrt{ }$ & $\sqrt{ }$ & $\sqrt{ }$ & $\sqrt{ }$ & $\sqrt{ }$ & $\sqrt{ }$ & $x$ & $x$ & $x$ & $x$ & $x$ \\
\hline 8 & Hou (72) & 30 & 30 & 46.12 & 46.78 & $\begin{array}{l}\text { seld-made Formula\&Bigu\&a- } \\
\text { Glucosidase }\end{array}$ & Bigu\&a-Glucosidase & 8 & $\sqrt{ }$ & $\sqrt{ }$ & $\sqrt{ }$ & $\sqrt{ }$ & $\sqrt{ }$ & $\sqrt{ }$ & $\sqrt{ }$ & $\sqrt{ }$ & $\sqrt{ }$ & $\sqrt{ }$ & $x$ & $\sqrt{ }$ \\
\hline 9 & Li (25) & 45 & 45 & 69.76 & 69.44 & Chinese medicine diet therapy & No treatment & 12 & $\sqrt{ }$ & $\sqrt{ }$ & $x$ & $\sqrt{ }$ & $\sqrt{ }$ & $\sqrt{ }$ & $\sqrt{ }$ & $x$ & $x$ & $x$ & $x$ & $x$ \\
\hline 10 & Li (32) & 30 & 30 & NS & NS & SMF\&Bigu\&a-Glucosidase & Bigu\&a-Glucosidase & 4 & $\sqrt{ }$ & $\sqrt{ }$ & $\sqrt{ }$ & $x$ & $x$ & $x$ & $x$ & $x$ & $x$ & $x$ & $x$ & $\sqrt{ }$ \\
\hline 11 & Li et al. (71) & 102 & 100 & 57.20 & 58.46 & Jiangtang Capsule\&Sulf & Sulf & 4 & $\sqrt{ }$ & $\sqrt{ }$ & $x$ & $\sqrt{ }$ & $\sqrt{ }$ & $\sqrt{ }$ & $\sqrt{ }$ & $x$ & $x$ & $x$ & $x$ & $x$ \\
\hline 12 & $\operatorname{Lin}(40)$ & 20 & 20 & 56.2 & 54.8 & Zhibodihuang Formula\&Bigu & Bigu & 4 & $\sqrt{ }$ & $\sqrt{ }$ & $\sqrt{ }$ & $x$ & $x$ & $x$ & $x$ & $x$ & $x$ & $x$ & $x$ & $x$ \\
\hline 13 & Liu et al. (70) & 29 & 29 & 46.5 & 46.9 & SMF\&Bigu & Bigu & 12 & $\sqrt{ }$ & $x$ & $\sqrt{ }$ & $\sqrt{ }$ & $\sqrt{ }$ & $\sqrt{ }$ & $\sqrt{ }$ & $x$ & $x$ & $x$ & $x$ & $x$ \\
\hline 14 & Liu et al. (69) & 30 & 30 & NS & NS & SMF\&DPP4 & DPP4 & 12 & $\sqrt{ }$ & $\sqrt{ }$ & $x$ & $x$ & $x$ & $x$ & $x$ & $x$ & $\sqrt{ }$ & $x$ & $x$ & $x$ \\
\hline 15 & Liu (29) & 30 & 30 & 57.3 & 56.7 & SMF\&Bigu\&Sulf & Bigu\&Sulf & 12 & $\sqrt{ }$ & $\sqrt{ }$ & $\sqrt{ }$ & $\sqrt{ }$ & $\sqrt{ }$ & $\sqrt{ }$ & $\sqrt{ }$ & $x$ & $x$ & $x$ & $x$ & $\sqrt{ }$ \\
\hline 16 & Lou and Zhao (68) & 52 & 50 & 53.5 & 54 & SMF\&Bigu & Bigu & 8 & $\sqrt{ }$ & $\sqrt{ }$ & $\sqrt{ }$ & $x$ & $x$ & $x$ & $x$ & $x$ & $x$ & $x$ & $x$ & $x$ \\
\hline 17 & Lv et al (35) & 60 & 60 & 54.61 & 55.77 & Yuye Formula\&insulin & insulin & 4 & $\sqrt{ }$ & $\sqrt{ }$ & $x$ & $\sqrt{ }$ & $\sqrt{ }$ & $\sqrt{ }$ & $\sqrt{ }$ & $x$ & $x$ & $x$ & $x$ & $x$ \\
\hline 18 & Lv et al. (67) & 45 & 30 & 58.9 & 58.8 & SMF\&a-Glucosidase & a-Glucosidase & 4 & $\sqrt{ }$ & $\sqrt{ }$ & $\sqrt{ }$ & $x$ & $x$ & $x$ & $x$ & $x$ & $x$ & $x$ & $x$ & $x$ \\
\hline 19 & Peng et al. (66) & 25 & 25 & 57 & 58 & SMF\&Bigu\&a-Glucosidase & Bigu\&a-Glucosidase & 8 & $\sqrt{ }$ & $\sqrt{ }$ & $x$ & $\sqrt{ }$ & $\sqrt{ }$ & $\sqrt{ }$ & $\sqrt{ }$ & $x$ & $x$ & $x$ & $x$ & $x$ \\
\hline 20 & Peng and $\mathrm{Xu}(36)$ & 68 & 68 & 74.86 & 75.01 & $\begin{array}{l}\text { Shenqi JiangTang Pill\&a- } \\
\text { Glucosidase }\end{array}$ & a-Glucosidase & 7 & $\sqrt{ }$ & $\sqrt{ }$ & $\times$ & $x$ & $x$ & $x$ & $x$ & $x$ & $x$ & $x$ & $x$ & $x$ \\
\hline 21 & Shang et al. (65) & 30 & 30 & 48.36 & 47.97 & SMF\&CSII & CSII & 8 & $x$ & $x$ & $x$ & $x$ & $x$ & $x$ & $x$ & $x$ & $\sqrt{ }$ & $\sqrt{ }$ & $x$ & $x$ \\
\hline 22 & Tang and $\mathrm{Li}(64)$ & 69 & 69 & 51.6 & 50.4 & Liuweidihuang Pill\&Bigu & Bigu & 12 & $\sqrt{ }$ & $\sqrt{ }$ & $\sqrt{ }$ & $\sqrt{ }$ & $\sqrt{ }$ & $\sqrt{ }$ & $\sqrt{ }$ & $x$ & $\sqrt{ }$ & $\sqrt{ }$ & $x$ & $\sqrt{ }$ \\
\hline 23 & $\begin{array}{l}\text { Wang and Wang } \\
\text { (27) }\end{array}$ & 29 & 27 & 59.1 & 59.2 & SMF\&Bigu\&Sulf & Bigu\&Sulf & 8 & $\sqrt{ }$ & $\sqrt{ }$ & $\sqrt{ }$ & $\sqrt{ }$ & $\sqrt{ }$ & $\sqrt{ }$ & $\sqrt{ }$ & $x$ & $x$ & $x$ & $\sqrt{ }$ & $x$ \\
\hline 24 & Wang (37) & 30 & 30 & 68.2 & 67.3 & SMF\&CSII & CSII & 2 & $\sqrt{ }$ & $x$ & $\sqrt{ }$ & $\sqrt{ }$ & $\sqrt{ }$ & $\sqrt{ }$ & $\sqrt{ }$ & $x$ & $x$ & $x$ & $x$ & $\sqrt{ }$ \\
\hline 25 & Wang (43) & 30 & 30 & 50.3 & 53.2 & Gankujiangtang Formula\&Bigu & Bigu & 12 & $\sqrt{ }$ & $\sqrt{ }$ & $\sqrt{ }$ & $\sqrt{ }$ & $\sqrt{ }$ & $\sqrt{ }$ & $\sqrt{ }$ & $x$ & $\sqrt{ }$ & $\sqrt{ }$ & $x$ & $\sqrt{ }$ \\
\hline 26 & Wang (33) & 30 & 30 & 50 & 53.7 & Zhibodihuang Formula\&Sulf & Sulf & 4 & $\sqrt{ }$ & $\sqrt{ }$ & $x$ & $x$ & $x$ & $x$ & $x$ & $x$ & $\sqrt{ }$ & $\sqrt{ }$ & $\sqrt{ }$ & $x$ \\
\hline 27 & Wu (28) & 20 & 18 & 51.85 & 54.78 & SMF\&Bigu & Bigu & 8 & $\sqrt{ }$ & $\sqrt{ }$ & $\sqrt{ }$ & $\sqrt{ }$ & $\sqrt{ }$ & $x$ & $x$ & $\sqrt{ }$ & $x$ & $x$ & $\sqrt{ }$ & $\sqrt{ }$ \\
\hline 28 & Xie and Lu (63) & 135 & 133 & $35-64$ & $36-60$ & Dihuangjiangtang pill\&Bigu & Bigu & 12 & $x$ & $x$ & $x$ & $\sqrt{ }$ & $\sqrt{ }$ & $\sqrt{ }$ & $\sqrt{ }$ & $x$ & $x$ & $x$ & $\times$ & $x$ \\
\hline 29 & Xu et al. (62) & 20 & 20 & 54 & 55 & Shenlinbaizhu Formula\&insulin & insulin & 12 & $x$ & $x$ & $x$ & $x$ & $x$ & $x$ & $x$ & $\sqrt{ }$ & $x$ & $x$ & $x$ & $x$ \\
\hline 30 & Yang (39) & 30 & 30 & NS & NS & Qianwenwu Formula\&Bigu & Bigu & 9 & $\sqrt{ }$ & $\sqrt{ }$ & $\sqrt{ }$ & $\sqrt{ }$ & $\sqrt{ }$ & $\sqrt{ }$ & $\sqrt{ }$ & $x$ & $x$ & $x$ & $x$ & $x$ \\
\hline 31 & Yu et al. (60) & 40 & 40 & 56.2 & 55.4 & Taipingtangke Pill\&Bigu & Bigu & 12 & $\sqrt{ }$ & $\sqrt{ }$ & $\sqrt{ }$ & $x$ & $x$ & $x$ & $x$ & $x$ & $x$ & $x$ & $x$ & $x$ \\
\hline 32 & Yu et al. (61) & 30 & 30 & $32-64$ & $34-67$ & SMF\&Bigu & Bigu & 8 & $\sqrt{ }$ & $\sqrt{ }$ & $x$ & $x$ & $x$ & $x$ & $x$ & $x$ & $x$ & $x$ & $\times$ & $x$ \\
\hline 33 & Zhang et al. (59) & 30 & 30 & 46.4 & 47.3 & SMF\&Bigu & Bigu & 4 & $\sqrt{ }$ & $\sqrt{ }$ & $\sqrt{ }$ & $x$ & $x$ & $x$ & $x$ & $x$ & $x$ & $x$ & $\times$ & $x$ \\
\hline 34 & Zhang et al. (52) & 32 & 30 & 54.4 & 56.5 & SMF\&Bigu & Bigu & 8 & $\sqrt{ }$ & $\sqrt{ }$ & $\sqrt{ }$ & $x$ & $x$ & $x$ & $x$ & $x$ & $x$ & $x$ & $x$ & $\sqrt{ }$ \\
\hline 35 & Zhang et al. (57) & 36 & 20 & $42-59$ & $44-57$ & SMF\&Bigu & Bigu & 4 & $\sqrt{ }$ & $\sqrt{ }$ & $\sqrt{ }$ & $x$ & $x$ & $x$ & $x$ & $x$ & $x$ & $x$ & $x$ & $x$ \\
\hline 36 & Zhang et al. (58) & 100 & 50 & 52.6 & 50.5 & SMF\&Bigu\&Sulf & Bigu\&Sulf & 8 & $\sqrt{ }$ & $\sqrt{ }$ & $x$ & $\sqrt{ }$ & $\sqrt{ }$ & $\sqrt{ }$ & $\sqrt{ }$ & $x$ & $\sqrt{ }$ & $x$ & $x$ & $\sqrt{ }$ \\
\hline 37 & Zhang and Pan (56) & 120 & 60 & 46.5 & 46.4 & Shendishengjin Capsule\&Sulf & Sulf & 8 & $\sqrt{ }$ & $\sqrt{ }$ & $x$ & $\sqrt{ }$ & $\sqrt{ }$ & $\sqrt{ }$ & $\sqrt{ }$ & $x$ & $x$ & $x$ & $x$ & $\sqrt{ }$ \\
\hline 38 & Zhang (41) & 30 & 30 & NS & NS & SMF\&Bigu\&a-Glucosidase & Bigu\&a-Glucosidase & 8 & $\sqrt{ }$ & $\sqrt{ }$ & $\sqrt{ }$ & $x$ & $x$ & $x$ & $x$ & $x$ & $x$ & $x$ & $x$ & $\sqrt{ }$ \\
\hline 39 & Zhang (38) & 32 & 31 & 54 & 56 & SMF\&Bigu\&a-Glucosidase & Bigu\&a-Glucosidase & 12 & $\sqrt{ }$ & $\sqrt{ }$ & $\sqrt{ }$ & $x$ & $x$ & $x$ & $x$ & $x$ & $x$ & $x$ & $x$ & $x$ \\
\hline 40 & Zhang (26) & 34 & 32 & 51 & 49 & self-made Formula & No treatment & 4 & $\sqrt{ }$ & $\sqrt{ }$ & $x$ & $x$ & $x$ & $x$ & $x$ & $x$ & $x$ & $x$ & $x$ & $\sqrt{ }$ \\
\hline 41 & Zhang (34) & 48 & 44 & 52.3 & 54.2 & Jiaweishenqidihuang Formula\&Bigu & Bigu & 12 & $\sqrt{ }$ & $x$ & $\sqrt{ }$ & $\sqrt{ }$ & $\sqrt{ }$ & $\sqrt{ }$ & $\sqrt{ }$ & $\sqrt{ }$ & $\sqrt{ }$ & $\sqrt{ }$ & $x$ & $x$ \\
\hline
\end{tabular}




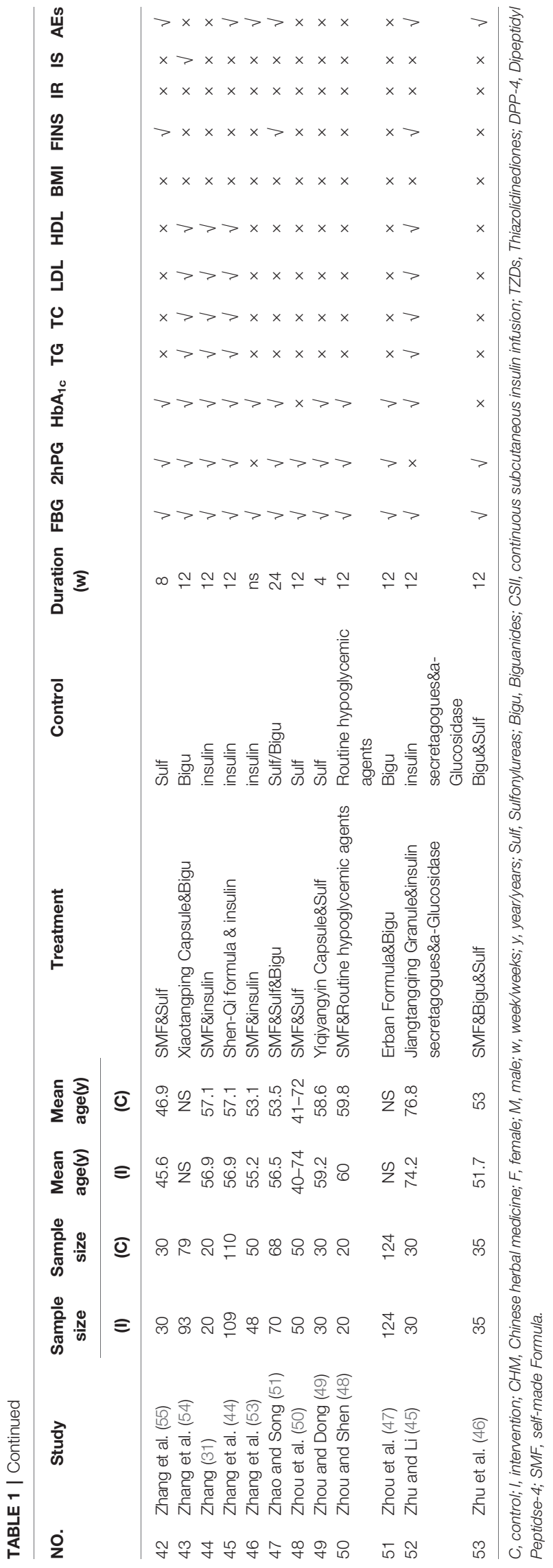

included in the methods section $(38,50,64)$. Publication bias was not detected (Egger's test, $\mathrm{t}=-0.71, P=0.48$ ). The overall methodological quality of the included studies was moderate.

\section{Effects of Intervention CHM Plus Hypoglycaemic Agents Versus Hypoglycaemic Agents}

Fasting Blood Glucose. Forty-eight RCTs including 4,375 participants assessed the effects of CHM plus hypoglycemic agents versus hypoglycemic agents alone; these studies used the same hypoglycemic agents in both groups (27-61, 64, 66-77). Treatment duration ranged from 2 to 24 weeks. Different classes of hypoglycemic agents were used across studies including biguanides, sulfonylureas, $\alpha$-Glucosidase inhibitors, DPP-4 inhibitors and insulins. Specific hypoglycemic agents include metformin, gliclazide, glibenclamide, gliquidone, glimepiride, acarbose, sitagliptin, and insulin.

The integrative use of CHM plus hypoglycemic agents was superior to hypoglycemic agents alone at reducing FBG levels at the end of treatment [MD $\left.-0.93(-1.10,-0.76) ; \mathrm{I}^{2}=84.2 \%\right]$, although heterogeneity was high. Meta-analysis of studies assessed as low risk of bias for sequence generation produced a similar result to the overall results with reduced heterogeneity [11 RCTs, 1,050 participants, MD $-0.68(-0.89,-0.47)$; $\mathrm{I}^{2}=$ 67.9\%] (31, 32, 34, 37, 38, 43, 44, 46, 64, 69, 73).

Subgroup analyses based on comparator drug class, patient age groups, baseline levels of FBG, CM syndrome differentiation, treatment duration, disease duration, and baseline levels of BMI all showed significant differences between groups (Table 2).

2-h Postprandial Blood Glucose. Forty-three RCTs including 4,004 participants assessed the effects of CHM plus hypoglycemic agents compared to hypoglycemic agents alone (27-33, 35, 36, 38-44, 46-52, 54-61, 64, 66-69, 71-77). All studies used the same hypoglycemic agent in both groups. Hypoglycemic agents included biguanides, sulfonylureas, $\alpha$-glucosidase inhibitors, DPP-4 inhibitors, and insulins. Specific agents include metformin, gliclazide, glibenclamide, glimepiride, gliquidone, glipizide, pioglitazone, acarbose, voglibose, sitagliptin, and insulin. Treatment duration ranged from 4 to 24 weeks.

Meta-analysis results showed that as integrative medicine, CHM plus hypoglycemic agents was superior to hypoglycemic agents alone at reducing $2 \mathrm{hPG}$ levels at the end of treatment [MD $\left.-1.46(-1.73,-1.20), \mathrm{I}^{2}=86.2 \%\right]$. Heterogeneity remained high after sensitivity analysis with studies with low risk of bias for sequence generation [nine studies, $\mathrm{n}=895, \mathrm{MD}-1.64$ $\left.(-2.27,-1.02) ; \mathrm{I}^{2}=91.8 \%\right](31,32,38,43,44,46,64,69,73)$ (Table 2).

Grouping of studies using metformin produced the biggest pool with 16 RCTs and 1,572 participants. The effect on 2hPG is similar to the overall result with reduced heterogeneity [MD $\left.-1.46(-1.73,-1.20) ; \mathrm{I}^{2}=44.3 \%\right](28,39,40,43,47,52,54,57$, $59-61,64,68,73,74,77)$. Combinations of different drug classes also showed a significant difference between groups, but heterogeneity remained high. Additional subgroup analyses based on patient age groups, baseline levels of FBG, CM 


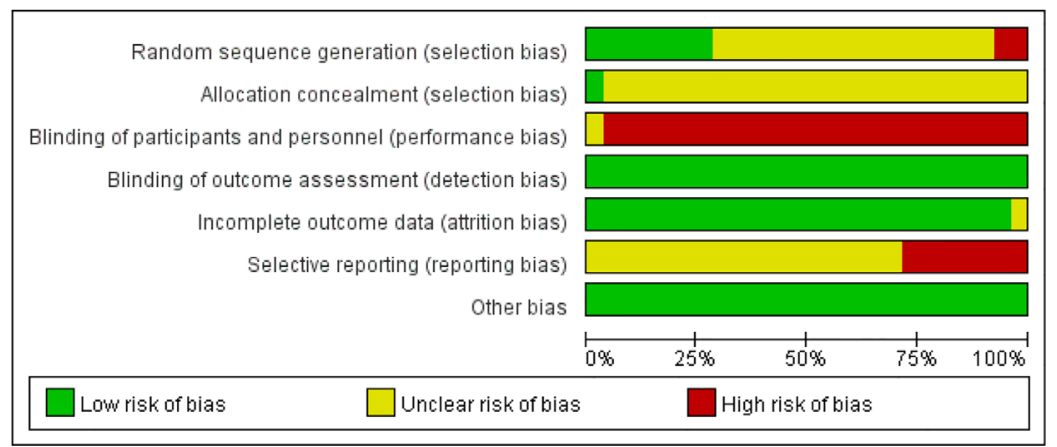

FIGURE 2 | Risk of bias of included studies.

syndrome differentiation, treatment duration, disease duration, and baseline levels of BMI all showed significant differences between groups (Table 2).

Hemoglobin A1c. Thirty-five RCTs including 3,009 participants assessed the effects of CHM plus hypoglycemic agents versus hypoglycemic agents alone $(27-32,34,37-45$, 47-49, 51-55, 57, 59, 60, 64, 67, 68, 70, 72-77). All studies used the same hypoglycemic agents in both groups, including biguanides, sulfonylureas, $\alpha$-glucosidase inhibitors, and insulins. Specific agents include metformin, gliclazide, glipizide, gliquidone, voglibose, acarbose, and insulin. Treatment duration ranged from 2 to 24 weeks.

CHM plus hypoglycemic agents was superior to hypoglycemic agents alone $\left[\mathrm{MD}-0.84(-1.05,-0.64), \mathrm{I}^{2}=\right.$ $88.2 \%$ ]. Heterogeneity remained high in subgroup analysis except in two subgroups where sulfonylureas or biguanides plus sulfonylureas were combined with CHM (Table 2). In two RCTs with 120 participants, the result indicated that the value of $\mathrm{HbA}_{1 \mathrm{c}}$ was reduced in people receiving CHM plus sulfonylureas compared to sulfonylureas alone $\left[\mathrm{MD}-0.65(-1.07,-0.24), \mathrm{I}^{2}=\right.$ 0.0\%] $(49,55)$. CHM plus biguanides and sulfonylureas were used as comparator in three RCTs, including 196 participants; the result showed that in the integrative medicine group, $\mathrm{HbA}_{1 \mathrm{c}}$ significantly lower compared to pharmacotherapy group alone [MD $\left.-0.35(-0.68,-0.02), \mathrm{I}^{2}=0.0 \%\right](27,29,75)$.

Blood Lipid Metabolism Indicators. Total Cholesterol. Twentyfour RCTs including 2,582 participants assessed the effects of $\mathrm{CHM}$ plus hypoglycemic agents versus hypoglycemic agents alone $(27-31,34,35,37,39,42-45,54,56,58,63,64,66,70-73$, 77). All studies used the same hypoglycemic agents in both groups; specific agents include metformin, glibenclamide, glipizide, glimepiride, acarbose, and insulin. Treatment duration ranged from 2 to 12 weeks.

Meta-analyses showed that CHM in addition to hypoglycemic agents was superior to hypoglycemic agents alone at reducing TG levels in T2DM patients [MD -0.40 $\left.(-0.51,-0.29), \mathrm{I}^{2}=79.2 \%\right]$. Meta-analysis of studies assessed as low risk for sequence generation produced a similar result that was more homogeneous [seven RCTs, 792 participants, MD $\left.-0.36(-0.50,-0.22) ; \mathrm{I}^{2}=50.5 \%\right](31,34,37,43,44,64,73)$.
Subgroup analyses by patient age groups, FBG level at baseline, disease duration and BMI levels at baseline showed similar effects on TG (Table 2). Combined use of CHM with biguanides, insulin, biguanides plus sulfonylureas and biguanides plus a-Glucosidase also produced significant difference between groups; results are like the overall analysis (Table 2).The combination of CHM to a sulfonylureas did not produce a better result than these agents alone $(56,71)$ (Table 2). In studies that provided details on CM syndrome differentiation, adding CHM to hypoglycemic agents showed more benefit in reducing TG levels in patients with $q i$ and yin deficiency $(29,31$, $34,35,37,39,71,77)$, yin deficiency with excessive heat $(43,64)$, but not those with spleen deficiency (28) (Table 2).

Triglyceride. Twenty-four RCTs including 2,582 participants assessed the effects of CHM plus hypoglycemic agents versus hypoglycemic agents alone $(27-31,34,35,37,39,42-45,54,56$, $58,63,64,66,70-73,77)$. All studies used the same hypoglycemic agents in both groups; specific agents include metformin, glibenclamide, glipizide, glimepiride, acarbose, and insulin. Treatment duration ranged from 2 to 12 weeks.

Meta-analyses showed that CHM in addition to hypoglycemic agents was superior to hypoglycemic agents alone at reducing TC levels in T2DM patients [MD -0.40 $\left.(-0.58,-0.22), \mathrm{I}^{2}=95.2 \%\right]$. Meta-analysis of studies assessed as low risk of bias for sequence generation showed no difference between groups [seven RCTs, 792 participants, MD $-0.34(-0.86$, 0.19); $\left.\mathrm{I}^{2}=98.3 \%\right](31,34,37,43,44,64,73)$.

Subgroup analysis showed that the combination of CHM with sulfonylureas $(56,71)$, insulin $(31,35,44)$, or biguanides plus sulfonylureas $(27,29,58)$ was not superior at reducing TC levels (Table 2). Studies with patient's age group of 41-61years (20 RCTs, $\mathrm{n}=2$,230) $(27-31,34,35,42-44,56,58,63,64,66,70-73$, 77) showed a significant difference between the two groups [MD $\left.-0.39(-0.59,-0.18) ; \mathrm{I}^{2}=96.0 \%\right]$ but not in the two studies with patients who are older $\left[\mathrm{n}=120, \mathrm{MD}-0.46(-0.99,0.08) ; \mathrm{I}^{2}=\right.$ $57.8 \%](37,45)$. Subgroup analyses by FBG level at baseline showed similar effects on TC. Analysis on studies that provided CM syndrome information showed that integrative medicine was superior to hypoglycemic agents alone in patients with qi and yin deficiency $(29,31,34,35,37,39,71,77)$, but not those with yin 


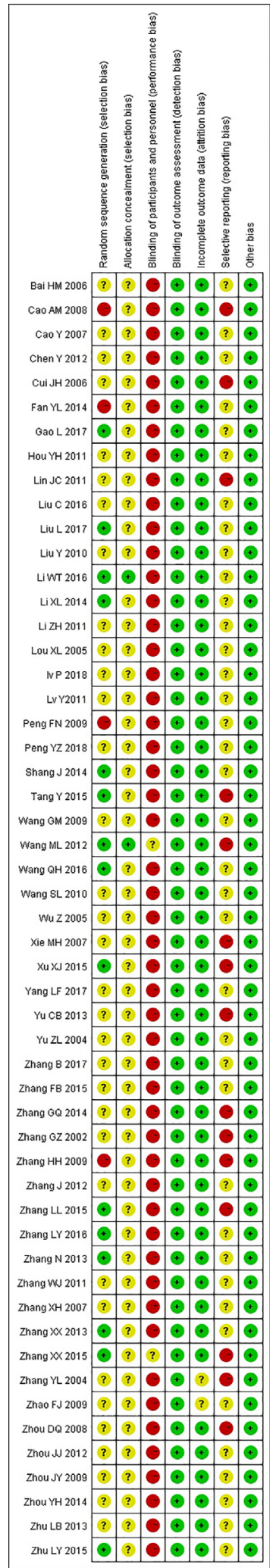

FIGURE 3 | Risk of bias summy of included studies. deficiency and excessive heat $(43,64)$ or spleen deficiency $(28)$ (Table 2). Studies with disease duration was $5-10$ years [six RCTs, $\mathrm{n}=612$, MD $\left.-0.63(-0.77,-0.50) ; \mathrm{I}^{2}=76.8 \%\right](29,54,66$, $70,71)$ showed a significant difference between the two groups but not in the study with disease duration of less than 5 years (27, $28,43,56,63,64,72)$ or equal or more than 10 years $(31,37,44$, $45,73,77$ ) disease duration subgroup (Table 2). Additional subgroup analyses by BMI level at baseline showed no difference between groups (Table 2).

Low Density Lipoprotein and High-Density Lipoprotein. Twenty-one RCTs including 2,338 participants assessed the effects of CHM plus hypoglycemic agents versus hypoglycemic agents alone $(27,29-31,34,35,37,39,43-45,54,56,58,63,64$, $66,70-73)$. Different classes of hypoglycemic agents were used, and treatment duration ranged from 2 to 12 weeks.

Meta-analysis of 21 studies showed that CHM plus hypoglycemic agents was superior to hypoglycemic agents alone at reducing LDL levels at the end of treatment $[\mathrm{MD}$ $\left.-0.45(-0.65,-0.27) ; \mathrm{I}^{2}=97.4 \%\right]$; however, heterogeneity was high. Seven studies were assessed as low risk of bias for sequence generation, subgroup analysis showed similar results to the overall studies $(31,34,37,43,44,64,73)$. Subgroup analysis by drug class showed that $\mathrm{CHM}$ added to sulfonylureas $(56,71)$ or insulin $(31,35,44)$ was no superior at reducing LDL levels compared to sulfonylureas or insulin alone (Table 2). Subgroup analyses by FBG level at baseline, disease duration or BMI level at baseline showed similar effects on LDL. Studies with age group of $41-64$ years showed significant between group results $(27,29-31$, $34,35,43,44,56,58,63,64,66,70-73)$. In studies that provided details on $\mathrm{CM}$ syndrome differentiation, subgroup analysis showed that CHM is better at reducing LDL levels in patients with $q i$ and $y i n$ deficiency $(29,31,34,35,37,39,71)$, but not in those with yin deficiency and excessive heat $(43,64)$ (Table 2).

Meta-analysis result of 21 studies showed that CHM plus hypoglycemic agents was superior to hypoglycemic agents alone at improving HDL levels [MD 0.15 (0.08, 0.22); $\mathrm{I}^{2}=93.9 \%$; however, heterogeneity was high. Results from studies with a low risk of bias for sequence generation produced a similar result (31, 34, 37, 43, 44, 64, 73) (Table 2). Subgroup analysis by drug class showed that CHM in combination with biguanides (34, 39, 43, $54,63,64,70,73)$, or insulin $(31,35,44)$ can improve HDL levels better than using the agents alone (Table 2). Studies with patient age group of 41-64 years showed significant between group results $(27,29-31,34,35,43,44,56,58,63,64,66,70-73)$ but not in two studies with older age groups $(37,47)$. Subgroup analysis using FBG level at baseline showed significant differences between groups with high heterogeneity (Table 2). In T2DM patients with $q i$ and yin deficiency $(29,31,34,35,37,39,71)$, integrative medicine was better at improving HDL levels than hypoglycemic agents alone (Table 2). Studies with disease duration was 5-10 years (five RCTs, $\mathrm{n}=542)(29,54,66,70$, 71) showed a significant difference between the two groups but not in studies with a disease duration of less than 5 years $(27,43$, $56,63,64,72)$ or more than 10 years $(31,37,44,45,73)$ (Table 2). Additional subgroup analyses by BMI level at baseline showed no difference between groups (Table 2). 
TABLE 2 | Summary of meta-analysis results and sub-group analysis.

\begin{tabular}{|c|c|c|c|c|c|c|}
\hline Treatment Vs. Comparison & Outcomes(unit) & Group & Subgroup & No. of Studies & $\mathrm{MD}[95 \% \mathrm{Cl}]$ & $\mathbf{I}^{2}$ \\
\hline \multirow{63}{*}{$\begin{array}{l}\text { CHM plus Hypoglycaemic } \\
\text { agents versus Hypoglycaemic } \\
\text { agents }\end{array}$} & FBG & All studies & All studies & $48(4,375)$ & $-0.93[-1.10,-0.76]^{\star}$ & $84.2 \%$ \\
\hline & $(\mathrm{mmol} / \mathrm{L})$ & Risk of bias SG & Low risk of bias SG & $11(1,050)$ & $-0.68[-0.89,-0.47]^{\star}$ & $67.9 \%$ \\
\hline & & comparator Drug class & Biguanides & $18(1,722)$ & $-0.91[-1.11,-0.70]^{\star}$ & $63.8 \%$ \\
\hline & & & Sulfonylureas & $7(770)$ & $-0.92[-1.58,-0.26]^{*}$ & $91.9 \%$ \\
\hline & & & Insulin & $4(477)$ & $-0.96[-1.57,-0.35]^{\star}$ & $85.0 \%$ \\
\hline & & & Biguanides + Sulfonylureas & $5(416)$ & $-1.18[-1.46,-0.89]^{\star}$ & $0.0 \%$ \\
\hline & & & Biguanides+ a-Glucosidase & $5(293)$ & $-0.97[-1.69,-0.24]^{\star}$ & $96.4 \%$ \\
\hline & & Age of patients & $41-64$ years & $36(3,219)$ & $-0.79[-1.02,-0.57]^{\star}$ & $86.7 \%$ \\
\hline & & & $>65$ years & $4(504)$ & $-1.28[-1.51,-1.06]^{\star}$ & $0.0 \%$ \\
\hline & & FBG level at baseline & $8-10 \mathrm{mmol} / \mathrm{L}$ & $27(2,413)$ & $-0.77[-0.95,-0.59]^{\star}$ & $74.9 \%$ \\
\hline & & & $\geq 10 \mathrm{mmol} / \mathrm{L}$ & $21(1,962)$ & $-1.15[-1.48,-0.82]^{\star}$ & $89.2 \%$ \\
\hline & & CM syndrome differentiation & qi and yin deficiency & $23(1,886)$ & $-0.97[-1.23,-0.72]^{\star}$ & $81.6 \%$ \\
\hline & & & yin deficiency and excessive heat & $6(430)$ & $-0.82[-1.05,-0.59]^{\star}$ & $23.0 \%$ \\
\hline & & & Spleen deficiency & $3(158)$ & $-0.57[-1.10,-0.04]^{\star}$ & $87.6 \%$ \\
\hline & & treatment duration & $\leq 3$ months & $46(4,139)$ & $-0.91[-1.08,-0.73]^{\star}$ & $83.9 \%$ \\
\hline & & & $\geq 6$ months & $1(138)$ & $-1.00[-1.34,-0.66]^{\star}$ & NA \\
\hline & & Disease duration & $<$ & $14(1,093)$ & $-0.80[-1.09,-0.51]^{\star}$ & $77.2 \%$ \\
\hline & & & $\geq 5-10$ years & 10(924) & $-1.07[-1.55,-0.59]^{\star}$ & $90.7 \%$ \\
\hline & & & $\geq 10$ years & $10(1,001)$ & $-0.88[-1.14,-0.62]^{\star}$ & $72.5 \%$ \\
\hline & & BMI level at baseline & $\geq 24-28 \mathrm{~kg} / \mathrm{m}^{2}$ & $3(193)$ & $-0.53[-0.87,-0.19]^{\star}$ & $0.0 \%$ \\
\hline & 2hPG & All studies & All studies & $43(4,004)$ & $-1.46[-1.73,-1.20]^{\star}$ & $86.2 \%$ \\
\hline & $(\mathrm{mmol} / \mathrm{L})$ & Risk of bias SG & Low risk of bias SG & 9(895) & $-1.64[-2.27,-1.02]^{\star}$ & $91.8 \%$ \\
\hline & & comparator Drug class & Biguanides & $16(1,572)$ & $-1.36[-1.62,-1.11]^{\star}$ & $44.3 \%$ \\
\hline & & & Sulfonylureas & $7(770)$ & $-1.60[-2.48,-0.72]^{\star}$ & $89.6 \%$ \\
\hline & & & Insulin & $3(379)$ & $-1.71[-3.37,-0.05]^{\star}$ & $91.2 \%$ \\
\hline & & & Biguanides + Sulfonylureas & $5(416)$ & $-1.12[-2.22,-0.02]^{\star}$ & $86.4 \%$ \\
\hline & & & Biguanides + a-Glucosidase & $5(298)$ & $-1.58[-2.41,-0.74]^{\star}$ & $94.7 \%$ \\
\hline & & Age of patients & 41-64 years & $33(2,968)$ & $-1.45[-1.78,-1.12]^{\star}$ & $86.7 \%$ \\
\hline & & & $>65$ years & $2(384)$ & $-1.65[-2.92,-0.39]^{\star}$ & $94.1 \%$ \\
\hline & & FBG level at baseline & $8-10 \mathrm{mmol} / \mathrm{L}$ & $24(2$ 200) & $-1.38[-1.74,-1.03]^{\star}$ & $85.6 \%$ \\
\hline & & & $\geq 10 \mathrm{mmol} / \mathrm{L}$ & $19(1,804)$ & $-1.56[-1.98,-1.15]^{\star}$ & $87.3 \%$ \\
\hline & & CM syndrome differentiation & qi and yin deficiency & $20(1,633)$ & $-1.25[-1.67,-0.84]^{\star}$ & $85.4 \%$ \\
\hline & & & yin deficiency and excessive heat & $6(430)$ & $-1.51[-2.16,-0.87]^{\star}$ & $71.4 \%$ \\
\hline & & & Spleen deficiency & $3(158)$ & $-0.96[-1.29,-0.63]^{\star}$ & $44.3 \%$ \\
\hline & & treatment duration & $\leq 3$ months & $42(3,866)$ & $-1.46[-1.73,-1.18]^{\star}$ & $86.3 \%$ \\
\hline & & & $\geq 6$ months & $1(138)$ & $-1.73[-2.07,-1.39]^{\star}$ & NA \\
\hline & & Disease duration & $<5$ years & $14(1,093)$ & $-1.57[-2.00,-1.15]^{\star}$ & $75.9 \%$ \\
\hline & & & $\geq 5-10$ years & $9(866)$ & $-1.35[-2.11,-0.59]^{\star}$ & $92.7 \%$ \\
\hline & & & $\geq 10$ years & $7(783)$ & $-1.38[-2.16,-0.61]^{\star}$ & $92.4 \%$ \\
\hline & & BMI level at baseline & $\geq 24-28 \mathrm{~kg} / \mathrm{m}^{2}$ & 2(98) & $-1.35[-2.25,-0.46]^{\star}$ & $46.6 \%$ \\
\hline & $\mathrm{HbA}_{1 \mathrm{c}}$ & All studies & All studies & $35(3,009)$ & $-0.84[-1.05,-0.64]^{\star}$ & $88.2 \%$ \\
\hline & $(\%)$ & Risk of bias SG & Low risk of bias SG & $9(920)$ & $-0.74[-1.16,-0.32]^{\star}$ & $93.9 \%$ \\
\hline & & comparator Drug class & Biguanides & $17(1,665)$ & $-0.85[-1.04,-0.66]^{\star}$ & $75.1 \%$ \\
\hline & & & Sulfonylureas & $2(120)$ & $-.65[-1.07,-0.24]^{\star}$ & $0.0 \%$ \\
\hline & & & Insulin & $3(357)$ & $-1.43[-2.69,-0.18]^{\star}$ & $94.8 \%$ \\
\hline & & & Biguanides + Sulfonylureas & $3(196)$ & $-0.35[-0.68,-0.02]^{\star}$ & $0.0 \%$ \\
\hline & & & Biguanides + a-Glucosidase & $4(248)$ & $-0.85[-1.68,-0.02]^{\star}$ & $96.7 \%$ \\
\hline & & Age of patients & $41-64$ years & $27(2,209)$ & $-0.77[-0.99,-0.55]^{\star}$ & $85.1 \%$ \\
\hline & & & $>65$ years & 3(368) & $-0.95[-1.76,-0.14]^{\star}$ & $77.2 \%$ \\
\hline & & FBG level at baseline & $8-10 \mathrm{mmol} / \mathrm{L}$ & $22(1,935)$ & $-0.73[-0.97,-0.50]^{\star}$ & $87.5 \%$ \\
\hline & & & $\geq 10 \mathrm{mmol} / \mathrm{L}$ & $13(1,074)$ & $-1.04[-1.41,-0.67]^{\star}$ & $86.9 \%$ \\
\hline & & CM syndrome differentiation & $q i$ and yin deficiency & $17(1,208)$ & $-0.67[-0.91,-0.44]^{\star}$ & $72.8 \%$ \\
\hline & & & yin deficiency and excessive heat & $4(300)$ & $-0.82[-1.21,-0.43]^{\star}$ & $69.3 \%$ \\
\hline & & & Spleen deficiency & $3(158)$ & $-1.07[-2.21,0.07]$ & $97.3 \%$ \\
\hline & & treatment duration & $\leq 3$ months & $33(2,773)$ & $-0.82[-1.04,-0.61]^{\star}$ & $88.5 \%$ \\
\hline & & & $\geq 6$ months & $1(138)$ & $-0.60[-0.99,-0.21]^{\star}$ & NA \\
\hline & & Disease duration & $<5$ years & $11(717)$ & $-0.72[-0.97,-0.47]^{\star}$ & $64.6 \%$ \\
\hline & & & $\geq 5-10$ years & $6(532)$ & $-0.91[-1.34,-0.48]^{\star}$ & $79.8 \%$ \\
\hline & & & $\geq 10$ years & 9(893) & $-0.90[-1.39,-0.42]^{\star}$ & $94.0 \%$ \\
\hline & & BMI level at baseline & $\geq 24-28 \mathrm{~kg} / \mathrm{m}^{2}$ & $3(193)$ & $-0.30[-0.62,0.02]$ & $39.9 \%$ \\
\hline & $\mathrm{TG}$ & All studies & All studies & $24(2,582)$ & $-0.40[-0.51,-0.29]^{\star}$ & $79.2 \%$ \\
\hline & $(\mathrm{mmol} / \mathrm{L})$ & Risk of bias SG & Low risk of bias SG & $7(792)$ & $-0.36[-0.50,-0.22]^{\star}$ & $50.5 \%$ \\
\hline & & comparator Drug class & Biguanides & $10(1,205)$ & $-0.39[-0.53,-0.24]^{\star}$ & $65.5 \%$ \\
\hline
\end{tabular}


TABLE 2 | Continued

\begin{tabular}{|c|c|c|c|c|c|c|}
\hline Treatment Vs. Comparison & Outcomes(unit) & Group & Subgroup & No. of Studies & $\mathrm{MD}[95 \% \mathrm{Cl}]$ & $\mathbf{I}^{2}$ \\
\hline & & & Sulfonylureas & $2(382)$ & $-0.06[-0.12,0.00]$ & $0.0 \%$ \\
\hline & & & Insulin & $3(379)$ & $-0.46[-0.74,-0.18]^{\star}$ & $56.5 \%$ \\
\hline & & & Biguanides + Sulfonylureas & $3(266)$ & $-0.37[-0.58,-0.17]^{\star}$ & $40.0 \%$ \\
\hline & & & Biguanides + a-Glucosidase & $2(110)$ & $-0.37[-0.52,-0.21]^{\star}$ & $0.0 \%$ \\
\hline & & \multirow[t]{2}{*}{ Age of patients } & $41-64$ years & $20(2,230)$ & $-0.37[-0.48,-0.26]^{\star}$ & $73.3 \%$ \\
\hline & & & $>65$ years & $2(120)$ & $-0.59[-0.81,-0.37]^{\star}$ & $0.0 \%$ \\
\hline & & \multirow[t]{2}{*}{ FBG level at baseline } & $8-10 \mathrm{mmol} / \mathrm{L}$ & $13(1,342)$ & $-0.36[-0.45,-0.27]^{\star}$ & $32.1 \%$ \\
\hline & & & $\geq 10 \mathrm{mmol} / \mathrm{L}$ & 10(972) & $-0.43[-0.64,-0.22]^{\star}$ & $88.1 \%$ \\
\hline & & \multirow[t]{3}{*}{ CM syndrome differentiation } & qi and yin deficiency & $8(773)$ & $-0.34[-0.52,-0.15]^{\star}$ & $76.1 \%$ \\
\hline & & & yin deficiency and excessive heat & $2(198)$ & $-0.19[-0.33,-0.05]^{\star}$ & $0.0 \%$ \\
\hline & & & Spleen deficiency & $1(38)$ & $-0.85[-1.73,0.03]$ & NA \\
\hline & & \multirow[t]{3}{*}{ Disease duration } & $<5$ years & $7(800)$ & $-0.31[-0.45,-0.18]^{\star}$ & $48.3 \%$ \\
\hline & & & $\geq 5-10 y e a r s$ & 6(612) & $-0.49[-0.81,-0.17]^{\star}$ & $92.7 \%$ \\
\hline & & & $\geq 10$ years & 6(695) & $-0.49[-0.62,-0.37]^{\star}$ & $6.8 \%$ \\
\hline & & BMI level at baseline & $\geq 24-28 \mathrm{~kg} / \mathrm{m}^{2}$ & $3(193)$ & $-0.57[-0.87,-0.28]^{\star}$ & $44.1 \%$ \\
\hline & \multirow{18}{*}{$\begin{array}{l}\mathrm{TC} \\
(\mathrm{mmol} / \mathrm{L})\end{array}$} & All studies & All studies & $24(2,582)$ & $-0.40[-0.58,-0.22]^{\star}$ & $95.2 \%$ \\
\hline & & Risk of bias SG & Low risk of bias SG & $7(792)$ & $-0.34[-0.86,0.19]$ & $98.3 \%$ \\
\hline & & \multirow[t]{5}{*}{ comparator Drug class } & Biguanides & $10(1,205)$ & $-0.36[-0.70,-0.02]^{\star}$ & $97.6 \%$ \\
\hline & & & Sulfonylureas & 2(382) & $-0.28[-0.82,0.25]$ & $89.1 \%$ \\
\hline & & & Insulin & $3(379)$ & $-0.13[-0.60,0.34]$ & $77.1 \%$ \\
\hline & & & Biguanides + Sulfonylureas & $3(266)$ & $-0.69[-1.59,0.21]$ & $73.9 \%$ \\
\hline & & & Biguanides + a-Glucosidase & $2(110)$ & $-0.44[-0.74,-0.15]^{\star}$ & $0.0 \%$ \\
\hline & & \multirow[t]{2}{*}{ Age of patients } & $41-64$ years & $20(2,230)$ & $-0.39[-0.59,-0.18]^{\star}$ & $96.0 \%$ \\
\hline & & & $>65$ years & 2(120) & $-0.46[-0.99,0.08]$ & $57.8 \%$ \\
\hline & & \multirow[t]{2}{*}{ FBG level at baseline } & $8-10 \mathrm{mmol} / \mathrm{L}$ & $13(1,342)$ & $-0.39[-0.70,-0.08]^{\star}$ & $97.0 \%$ \\
\hline & & & $\geq 10 \mathrm{mmol} / \mathrm{L}$ & 10(972) & $-0.50[-0.66,-0.34]^{*}$ & $66.5 \%$ \\
\hline & & \multirow[t]{3}{*}{ CM syndrome differentiation } & qi and yin deficiency & $8(773)$ & $-0.40[-0.67,-0.12]$ * & $95.9 \%$ \\
\hline & & & yin deficiency and excessive heat & 2(198) & $-0.77[-1.92,0.38]$ & $98.7 \%$ \\
\hline & & & Spleen deficiency & $1(38)$ & $0.12[-0.38,0.62]$ & NA \\
\hline & & \multirow[t]{3}{*}{ Disease duration } & $<5$ years & $7(800)$ & $-0.39[-0.94,0.16]$ & $96.0 \%$ \\
\hline & & & $\geq 5-10$ years & $6(612)$ & $-0.63[-0.77,-0.50]^{\star}$ & $76.8 \%$ \\
\hline & & & $\geq 10$ years & 6(695) & $-0.16[-0.43,0.11]$ & $71.3 \%$ \\
\hline & & BMI level at baseline & $\geq 24-28 \mathrm{~kg} / \mathrm{m}^{2}$ & $3(193)$ & $-0.02[-0.15,0.10]$ & $10.6 \%$ \\
\hline & \multirow{17}{*}{$\begin{array}{l}\text { LDL } \\
(\mathrm{mmol} / \mathrm{L})\end{array}$} & All & All studies & $21(2,338)$ & $-0.45[-0.65,-0.27]^{*}$ & $97.4 \%$ \\
\hline & & Risk of bias SG & Low risk of bias SG & $7(792)$ & $-0.68[-1.10,-0.26]^{\star}$ & $97.9 \%$ \\
\hline & & \multirow[t]{5}{*}{ comparator Drug class } & Biguanides & $8(1,031)$ & $-0.51[-0.88,-0.14]^{\star}$ & $98.5 \%$ \\
\hline & & & Sulfonylureas & 2(382) & $-0.06[-0.16,0.04]$ & $0.0 \%$ \\
\hline & & & Insulin & $3(379)$ & $-0.57[-1.18,0.05]$ & $97.6 \%$ \\
\hline & & & Biguanides + Sulfonylureas & $3(266)$ & $-0.45[-0.60,-0.30]^{\star}$ & $0.0 \%$ \\
\hline & & & Biguanides + a-Glucosidase & $2(110)$ & $-0.39[-0.66,-0.12]^{\star}$ & $78.1 \%$ \\
\hline & & \multirow[t]{2}{*}{ Age of patients } & $41-64$ years & $17(1,986)$ & $-0.45[-0.69,-0.21]^{\star}$ & $97.7 \%$ \\
\hline & & & $>65$ years & $2(120)$ & $-0.73[-1.82,0.35]$ & $78.9 \%$ \\
\hline & & \multirow[t]{2}{*}{ FBG level at baseline } & $8-10 \mathrm{mmol} / \mathrm{L}$ & $12(1,206)$ & $-0.53[-0.83,-0.22]^{\star}$ & $97.7 \%$ \\
\hline & & & $\geq 10 \mathrm{mmol} / \mathrm{L}$ & $8(864)$ & $-0.23[-0.33,-0.13]^{\star}$ & $68.4 \%$ \\
\hline & & CM syndrome differentiation & qi and yin deficiency & $7(637)$ & $-0.29[-0.44,-0.15]^{\star}$ & $75.1 \%$ \\
\hline & & & yin deficiency and excessive heat & 2(198) & $-0.79[-2.03,0.46]$ & $99.4 \%$ \\
\hline & & Disease duration & $<5$ years & $6(762)$ & $-0.59[-1.08,-0.09]^{\star}$ & $98.8 \%$ \\
\hline & & & $\geq 5-10$ years & $5(542)$ & $-0.31[-0.46,-0.16]^{\star}$ & $80.7 \%$ \\
\hline & & & $\geq 10$ years & $5(559)$ & $-0.64[-1.11,-0.17]^{\star}$ & $95.5 \%$ \\
\hline & & BMI level at baseline & $\geq 24-28 \mathrm{~kg} / \mathrm{m}^{2}$ & $2(155)$ & $-0.27[-0.35,-0.20]^{\star}$ & $0.0 \%$ \\
\hline & HDL & All & All studies & $21(2,338)$ & $0.15[0.08,0.22]^{*}$ & $93.9 \%$ \\
\hline & $(\mathrm{mmol} / \mathrm{L})$ & Risk of bias SG & Low risk of bias SG & $7(792)$ & $0.18[0.07,0.29]^{*}$ & $93.3 \%$ \\
\hline & & comparator Drug class & Biguanides & $8(1,031)$ & $0.24[0.14,0.33]^{\star}$ & $89.6 \%$ \\
\hline & & & Sulfonylureas & $2(382)$ & $0.18[-0.21,0.58]$ & $96.8 \%$ \\
\hline & & & Insulin & 3(379) & $0.20[0.05,0.34]^{\star}$ & $93.6 \%$ \\
\hline & & & Biguanides + Sulfonylureas & $3(266)$ & $0.02[-0.17,0.21]$ & $81.0 \%$ \\
\hline & & & Biguanides + a-Glucosidase & $2(110)$ & $-0.04[-0.12,0.04]$ & $21.1 \%$ \\
\hline & & Age of patients & $41-64$ years & $17(1986)$ & $0.16[0.08,0.25]^{*}$ & $94.6 \%$ \\
\hline & & & $>65$ years & $2(120)$ & $0.01[-0.05,0.07]$ & $0.0 \%$ \\
\hline & & FBG level at baseline & $8-10 \mathrm{mmol} / \mathrm{L}$ & $12(1,206)$ & $0.13[0.04,0.22]^{\star}$ & $93.5 \%$ \\
\hline & & & $\geq 10 \mathrm{mmol} / \mathrm{L}$ & $8(864)$ & $0.12[0.02,0.22]^{\star}$ & $89.8 \%$ \\
\hline & & CM syndrome differentiation & qi and yin deficiency & $7(637)$ & $0.17[0.09,0.25]^{\star}$ & $75.5 \%$ \\
\hline & & & yin deficiency and excessive heat & 2(198) & $0.16[-0.04,0.35]$ & $82.7 \%$ \\
\hline
\end{tabular}


TABLE 2 | Continued

\begin{tabular}{|c|c|c|c|c|c|c|}
\hline Treatment Vs. Comparison & Outcomes(unit) & Group & Subgroup & No. of Studies & $\mathrm{MD}[95 \% \mathrm{Cl}]$ & $\mathbf{I}^{2}$ \\
\hline & & \multirow[t]{3}{*}{ Disease duration } & $<5$ years & $6(762)$ & $0.13[-0.04,0.31]$ & $94.3 \%$ \\
\hline & & & $\geq 5-10$ years & $5(542)$ & $0.17[0.07,0.27]^{\star}$ & $84.9 \%$ \\
\hline & & & $\geq 10$ years & $5(559)$ & $0.16[-0.00,0.33]$ & $95.7 \%$ \\
\hline & & BMI level at baseline & $\geq 24-28 \mathrm{~kg} / \mathrm{m}^{2}$ & $2(155)$ & $0.03[-0.18,0.23]$ & $90.8 \%$ \\
\hline & \multirow{17}{*}{$(\mathrm{mU} / \mathrm{Lor} \mu \mathrm{IU} / \mathrm{ml})$} & All studies & All studies & $12(1,049)$ & $-1.03[-2.35,0.29]$ & $86.9 \%$ \\
\hline & & Risk of bias SG & Low risk of bias SG & $5(413)$ & $-1.60[-4.42,1.21]$ & $92.3 \%$ \\
\hline & & comparator Drug class & Biguanides & $3(293)$ & $-2.89[-6.96,1.18]$ & $95.7 \%$ \\
\hline & & & Sulfonylureas & $3(228)$ & $-2.03[-3.21,-0.84]^{\star}$ & $0.0 \%$ \\
\hline & & & Biguanides + Sulfonylureas & $1(150)$ & $0.60[-1.16,2.36]$ & NA \\
\hline & & & Biguanides + a-Glucosidase & $1(60)$ & $-2.10[-2.79,-1.41]$ & NA \\
\hline & & Age of patients & $41-64$ years & 10(929) & $-1.46[-2.85,-0.07]^{*}$ & $88.2 \%$ \\
\hline & & & $>65$ years & $1(60)$ & $1.32[-2.11,4.75]$ & NA \\
\hline & & FBG level at baseline & $8-10 \mathrm{mmol} / \mathrm{L}$ & $8(641)$ & $-1.95[-3.45,-0.45]^{\star}$ & $87.4 \%$ \\
\hline & & & $\geq 10 \mathrm{mmol} / \mathrm{L}$ & $3(348)$ & $1.62[0.09,3.15]$ & $32.2 \%$ \\
\hline & & CM syndrome differentiation & qi and yin deficiency & $3(215)$ & $0.17[-2.08,2.42]$ & $47.4 \%$ \\
\hline & & & yin deficiency and excessive heat & $3(258)$ & $-3.43[-7.37,0.49]$ & $95.3 \%$ \\
\hline & & treatment duration & $\leq 3$ months & $11(911)$ & $-1.41[-2.68,-0.15]^{\star}$ & $84.5 \%$ \\
\hline & & & $\geq 6$ months & $1(138)$ & $2.85[0.96,4.73]$ & NA \\
\hline & & Disease duration & $<5$ years & $5(378)$ & $-2.96[-4.93,-0.99]$ & $90.7 \%$ \\
\hline & & & $\geq 10$ years & $2(168)$ & $-0.80[-4.34,2.74]$ & $70.1 \%$ \\
\hline & & BMI level at baseline & $\geq 24-28 \mathrm{~kg} / \mathrm{m}^{2}$ & $2(155)$ & $-1.19[-3.21,0.82]$ & $81.8 \%$ \\
\hline & \multirow[t]{11}{*}{$\mathbb{I R}$} & All studies & All studies & $7(581)$ & $-1.11[-1.44,-0.77]^{\star}$ & $55.0 \%$ \\
\hline & & Risk of bias SG & Low risk of bias SG & $4(353)$ & $-1.14[-1.67,-0.60]^{\star}$ & $72.6 \%$ \\
\hline & & comparator Drug class & Biguanides & $3(293)$ & $-0.80[-1.04,-0.56]^{\star}$ & $0.0 \%$ \\
\hline & & & Sulfonylureas & $2(168)$ & $-1.30[-1.85,-0.75]^{\star}$ & $0.0 \%$ \\
\hline & & & Biguanides + a-Glucosidase & $1(60)$ & $-0.98[-1.52,-0.44]^{\star}$ & NA \\
\hline & & FBG level at baseline & 8-10 mmol/L & $6(521)$ & $-0.91[-1.12,-0.69]^{\star}$ & $5.5 \%$ \\
\hline & & CM syndrome differentiation & qi and yin deficiency & $1(95)$ & $-0.90[-1.28,-0.52]$ & NA \\
\hline & & & yin deficiency and excessive heat & $3(258)$ & $-0.79[-1.10,-0.48]^{\star}$ & $3.5 \%$ \\
\hline & & Disease duration & $<5$ years & 4(318) & $-0.82[-1.08,-0.57]^{\star}$ & $0.0 \%$ \\
\hline & & & $\geq 10$ years & $1(108)$ & $-1.49[-2.23,-0.75]^{\star}$ & NA \\
\hline & & BMI level at baseline & $\geq 24-28 \mathrm{~kg} / \mathrm{m}^{2}$ & $2(155)$ & $-0.93[-1.24,-0.62]^{\star}$ & $0.0 \%$ \\
\hline & \multirow[t]{13}{*}{ IS } & All studies & All studies & $5(434)$ & $0.09[-0.26,0.43]$ & $95.0 \%$ \\
\hline & & comparator Drug class & Biguanides & $2(210)$ & $-0.14[-0.97,0.69]$ & $98.7 \%$ \\
\hline & & & Sulfonylureas & $2(168)$ & $0.17[0.05,0.28]^{\star}$ & $0.0 \%$ \\
\hline & & & Biguanides + Sulfonylureas & $1(56)$ & $0.56[0.05,1.07]^{\star}$ & NA \\
\hline & & Age of patients & 41-64 years & $4(262)$ & $0.23[0.12,0.34]^{\star}$ & $34.6 \%$ \\
\hline & & FBG level at baseline & $8-10 \mathrm{mmol} / \mathrm{L}$ & $3(224)$ & $0.19[0.03,0.36]^{*}$ & $35.4 \%$ \\
\hline & & & $\geq 10 \mathrm{mmol} / \mathrm{L}$ & $2(210)$ & $-0.14[-0.98,0.69]$ & $98.7 \%$ \\
\hline & & CM syndrome differentiation & yin deficiency and excessive heat & $1(60)$ & $0.21[0.06,0.36]^{\star}$ & NA \\
\hline & & & Spleen deficiency & $1(38)$ & $0.28[0.18,0.38]^{\star}$ & NA \\
\hline & & Disease duration & $<5$ years & $3(154)$ & $0.27[0.19,0.35]^{*}$ & $0.0 \%$ \\
\hline & & & $\geq 5-10$ years & $1(172)$ & $-0.57[-0.74,-0.40]$ & NA \\
\hline & & & $\geq 10$ years & $1(108)$ & $0.09[-0.11,0.29]$ & NA \\
\hline & & BMI level at baseline & $\geq 24-28 \mathrm{~kg} / \mathrm{m}^{2}$ & $1(38)$ & $0.28[0.18,0.38]^{\star}$ & NA \\
\hline & $\mathrm{BMl}$ & All studies & All studies & $4(233)$ & $-0.45[-0.99,0.08]$ & $9.4 \%$ \\
\hline & $\left(\mathrm{kg} / \mathrm{m}^{2}\right)$ & Risk of bias SG & Low risk of bias SG & $2(135)$ & $-0.38[-2.83,2.07]$ & $68.4 \%$ \\
\hline \multirow[t]{2}{*}{ CHM vs. lifestyle intervention } & $\begin{array}{l}\text { FBG } \\
(\mathrm{mmol} / \mathrm{L})\end{array}$ & All studies & All studies & $1(72)$ & $-0.62[-1.28,0.04]$ & NA \\
\hline & $\begin{array}{l}2 \mathrm{hPG} \\
(\mathrm{mmol} / \mathrm{L})\end{array}$ & All studies & All studies & $1(72)$ & $-2.14[-2.81,-1.47]^{\star}$ & NA \\
\hline \multirow[t]{4}{*}{$\begin{array}{l}\mathrm{CHM} \text { as food vs. Lifestyle } \\
\text { intervention }\end{array}$} & $\begin{array}{l}\text { FBG } \\
(\mathrm{mmol} / \mathrm{L})\end{array}$ & All studies & All studies & $1(90)$ & $-1.08[-1.86,-0.30]^{*}$ & NA \\
\hline & $\begin{array}{l}2 \mathrm{hPG} \\
(\mathrm{mmol} / \mathrm{L})\end{array}$ & All studies & All studies & $1(90)$ & $-1.23[-1.96,-0.50]^{\star}$ & NA \\
\hline & $\begin{array}{l}\text { TG } \\
(\mathrm{mmol} / \mathrm{L})\end{array}$ & All studies & All studies & $1(90)$ & $-0.30[-0.69,0.09]$ & NA \\
\hline & $\begin{array}{l}\mathrm{TC} \\
(\mathrm{mmol} / \mathrm{L})\end{array}$ & All studies & All studies & $1(90)$ & $-0.66[-1.10,-0.23]^{\star}$ & NA \\
\hline
\end{tabular}

*Statistically significant difference between groups.

Cl, confidence interval; CM, Chinese medicine; FBG, fasting blood glucose; 2hPG, 2-hour postprandial blood glucose; HbA1C, hemoglobin A1c; TG, triglyceride; TC, total cholesterol; LDL, Iow density lipoprotein; HDL, high-density lipoprotein; FINS, fasting insulin; IR, insulin resistance; IS, insulin resistance index; BMI, body mass index; MD, mean difference; SG, sequence generation. 
$\beta$-Cell Function Indicators. Fasting Insulin. Twelve RCTs including 1,049 participants assessed the effects of CHM plus hypoglycemic agents versus hypoglycemic agents alone $(33,34$, $43,45,51,55,58,64,65,69,72,76)$. Different classes of hypoglycemic agents were included and treatment duration ranged from 4 to 24 weeks.

At the end of treatment, CHM plus hypoglycemic agents was not superior to hypoglycemic agents alone at reducing FINS levels [MD $\left.-1.03(-2.34,0.29), \mathrm{I}^{2}=86.9 \%\right]$. Meta-analysis of results from studies with a low risk of bias for sequence generation showed similar results from the overall result $(34,43,64,65,69)$. Subgroup analysis by hypoglycemic agent class showed varied results for different class of drugs; there were benefits seen in the addition of $\mathrm{CHM}$ to sulfonylureas but not in other hypoglycaemic agents (Table 2). Studies with patient age group of 41-64 years showed significant difference between group results $(33,34,43,51,55,58$, $64,65,72,76$ ) but not in older subgroup (45). Subgroup analysis based on FBG level at baseline, the lower reading of $8-10 \mathrm{mmol} / \mathrm{L}$ subgroup $(33,34,43,55,64,69,72,76)$ showed more benefit but not in higher subgroup $(45,51,58)$. Studies with a shorter treatment duration of less than 3 months showed benefit in adding CHM to hypoglycaemic agents $(33,34,43,45,55,58,64,65,69,72,76)$, but not in studies with a longer treatment (51). Additional subgroup analyses by CM syndrome differentiation, disease duration and BMI level at baseline showed no difference between groups (Table 2).

Homeostatic model assessment of insulin resistance. Seven RCTs including 581 participants assessed the effects of CHM plus hypoglycemic agents versus hypoglycemic agents alone (33, $34,43,64,65,72,76)$. Hypoglycemic agents included biguanides, sulfonylureas, $\alpha$-glucosidase inhibitors, and insulins. Treatment duration ranged from 4 to 12 weeks.

CHM plus hypoglycemic agents was superior to hypoglycaemic agents alone $\left[\mathrm{MD}-1.11(-1.44,-0.77) ; \mathrm{I}^{2}=\right.$ $55.0 \%]$. Meta-analysis of results from studies with a low risk of bias for sequence generation showed similar results $(34,43,64$, 65); however, heterogeneity was high. Subgroup analyses based on drug class, baseline levels of FBG, disease duration and baseline levels of BMI all showed significant differences between groups (Table 2). Subgroup analysis by CM differentiation showed benefit of adding CHM to hypoglycemic group than hypoglycemic agents alone in patients with yin deficiency and excessive heat $(33,43,64)$ (Table 2).

Insulin resistance index. Five RCTs including 434 participants assessed the effects of CHM plus hypoglycemic agents versus hypoglycemic agents alone $(27,28,33,54,76)$. Various hypoglycemic agents were used including metformin, gliclazide, and glimepiride. All studies had a treatment duration of less than or equal to 12 weeks; the shortest treatment duration was 4 weeks.

Meta-analysis showed that CHM plus hypoglycemic agents was not superior to hypoglycemic agents alone [MD $0.09(-0.26$, $\left.0.43), \mathrm{I}^{2}=95.0 \%\right]$. Subgroup analysis by drug class showed benefit in adding CHM to sulfonylureas $(33,76)$ and the combination of biguanides with sulfonylureas (27). No significant difference was observed when CHM was added to biguanides $(28,54)$. Subgroup analysis using age of patients of 41-64 years showed benefit (27, $28,33,76)$. Subgroup analysis based on lower FBG level at baseline
$(8-10 \mathrm{mmol} / \mathrm{L})(27,33,76)$ showed more benefit but not in the higher subgroup $(28,54)$. In studies that provided information on CM differentiation, meta-analyses showed a benefit in adding $\mathrm{CHM}$ to hypoglycemic agents in patients with yin deficiency and excessive heat (33) or spleen deficiency (28). Studies with disease duration of less than 5 years (three RCTs, $\mathrm{n}=154)(27,28$, 33) showed a significant difference between the two groups but not those studies with more than 5 years of disease duration $(54,76)$. Additional subgroup analyses by BMI level at baseline showed difference between groups (28) (Table 2).

Body Mass Index. Four RCTs including 233 participants assessed the effects of CHM plus hypoglycemic agents versus hypoglycemic agents alone $(28,34,62,72)$. Hypoglycemic agents included biguanides, $\boldsymbol{\alpha}$-Glucosidase inhibitors and insulins. Specific agents include metformin, acarbose, and insulin. Treatment duration ranged from 8 to 12 weeks.

Meta-analysis results showed that $\mathrm{CHM}$ plus hypoglycemic agents was not superior to hypoglycemic agents alone at improving BMI $\left[\mathrm{MD}-0.45(-0.99,0.08) ; \mathrm{I}^{2}=9.4 \%\right)$. Metaanalysis of studies assessed as low risk for sequence generation produced a similar result [two studies, 135 participants, MD $\left.-0.38(-2.83,2.07) ; \mathrm{I}^{2}=68.4 \%\right](34,62)$, and no difference was found between groups.

\section{CHM Plus Lifestyle Intervention Versus Lifestyle Intervention Alone}

One RCT with 72 participants compared CHM (including Shanyao) plus lifestyle intervention with lifestyle intervention for 4 weeks (26). The result showed CHM together with lifestyle intervention was superior to lifestyle intervention alone in reducing $2 \mathrm{hPG}$ and $\mathrm{TC}$ levels at the end of treatment [MD $-2.14(-2.81,-1.47),-0.66(-1.10,-0.23)]$; there was no difference in the FBG and TG levels between the two groups [MD $-0.62(-1.28,0.04),-0.30(-0.69,0.09)]$.

\section{CHM Diet Therapy Plus Lifestyle Intervention Versus Lifestyle Intervention Alone}

One RCT ( $\mathrm{n}=90)$ compared CHM as diet therapy (including Shanyao) with lifestyle intervention with a treatment duration of 12 weeks (25). The result showed that combination of Chinese diet therapy using Shanyao and lifestyle intervention was superior to lifestyle intervention alone at reducing FBG and 2hPG levels at the end of treatment $[\mathrm{MD}-1.08(-1.86,-0.30),-1.23(-1.96,-0.50)]$.

\section{Adverse Events}

Out of the 53 studies, 21 studies reported on AEs. Of these, 10 studies provided specific details about the AEs.

CHM Plus Hypoglycaemic Agents vs. Hypoglycaemic Agents. In twenty RCTs of CHM plus hypoglycemic drugs versus hypoglycemic drugs, ten studies reported no AEs (28, 30, 32, $41,51,52,55,72,76,77)$, ten studies provided specific details about AEs $(29,37,43-46,53,56,58,64)$. In the integrative medicine group, the most common AEs were hypoglycemia (four cases), nausea (three cases), hypertension (two cases), insomnia (one case), epigastric discomfort (two cases), diarrhea (two cases), frequency of urine (three cases), drugrelated adverse reactions (one case unknown). There were three 
cases of diarrhea reported; however, it was not clear whether the AEs were from the treatment group or the control group (43).

Twenty-four AEs were reported in the hypoglycemic drug group. In the hypoglycemic agents group, hypoglycemia (seven cases) was the most common AE. Other AEs included nausea (three cases), headache (two cases), stomach distention (three cases), dyspepsia (two cases), fatigue (two cases), rash (one case), and four other AEs were not described in detail.

One study that compared CHM plus lifestyle intervention to lifestyle intervention reported no AEs (26).

\section{Assessment Using GRADE}

An assessment of the quality of the evidence from RCTs was undertaken using GRADE. Interventions, comparators, and outcomes included were selected based on a consensus process. Comparisons were: CHM plus hypoglycemic agents versus hypoglycemic agents, CHM plus lifestyle intervention versus lifestyle intervention and CHM diet therapy plus lifestyle intervention versus lifestyle intervention.

Evidence of Shanyao formulae for T2DM was low to moderate quality (Table 3 ). The results showed that oral

TABLE 3 | GRADE: Quality of the evidence of Shanyao formulae for T2DM.

\begin{tabular}{lcc}
\hline Outcomes & $\begin{array}{c}\text { № of participants } \\
\text { (studies)Follow-up }\end{array}$ & $\begin{array}{c}\text { Certainty of the } \\
\text { evidence(GRADE) }\end{array}$
\end{tabular}

CHM plus Hypoglycemic agents vs. Hypoglycemic agents

Fasting blood glucose (FBG)

Treatment duration: mean 8.85

weeks

2-hour Postprandial blood glucose

(2hPG)

Treatment duration: mean 8.79

weeks

Glycosylated Hemoglobin A1c

(HbA1c)

Treatment duration: mean 9.32

weeks

Triglyceride (TG)

Treatment duration: mean 9.04

weeks

Cholesterol (TC)

Treatment duration: mean 9.04

weeks

Fasting insulin (FINS)

Follow-up: range 1 to 30 weeks

Treatment duration: mean 10.67

weeks

CHM plus lifestyle intervention vs. lifestyle intervention

Fasting blood glucose (changed 72

from baseline)(FBG)

Treatment duration 4 weeks

2-hour Postprandial blood glucose

(changed from baseline)(2hPG)

Treatment duration 4 weeks

CHM diet therapy plus lifestyle intervention vs. lifestyle intervention

Fasting blood glucose (changed 90

Treatment duration 12 weeks

2-hour Postprandial blood glucose

(changed from baseline)(2hPG)

Treatment duration 12 weeks

Triglyceride(changed from baseline)

(TG)

Treatment duration 12 weeks

Cholesterol(changed from baseline)

(TC)

$\begin{array}{ll}4,375 & \bigoplus \bigoplus \bigoplus \bigcirc \\ \text { (48 RCTs) } & \text { MODERATE }{ }^{a} \\ 4,004 & \bigoplus \bigoplus \bigoplus \bigcirc \\ \text { (43 RCTs) } & \text { MODERATE }{ }^{\mathrm{a}}\end{array}$

\section{3,009}

(35 RCTs)

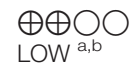

2,582

(24 RCTs)

\section{2,582}

(24 RCTs)

1,049

(12 RCTs)

$\oplus \mathrm{LOW}^{\mathrm{a}, \mathrm{b}}$
$\mathrm{P}$

MODERATE

(one RCT)

$\begin{array}{ll}72 & \oplus \oplus \oplus \bigcirc \\ \text { (one RCT) } & \text { MODERATE }\end{array}$

$\bigoplus \bigoplus \bigoplus \bigcirc$

(one RCT)

90

(one RCT)

90

(one RCT)

90

(one RCT) $\bigoplus \oplus \bigcirc \bigcirc$

LOW $^{a, b}$

$\oplus \oplus \oplus \circ$

MODERATE ${ }^{a}$

$\oplus \oplus \oplus \circ$

MODERATEC

$\oplus \oplus \oplus \circ$

MODERATE

Risk with [Hypoglycemic drugs]

The mean fasting blood glucose was $\mathbf{7 . 4 3}$ $\mathrm{mmol} / \mathrm{L}$

The mean postprandial blood glucose was $10.44 \mathrm{mmol} / \mathrm{L}$

The mean glycosylated Hemoglobin A1c was $7.44 \%$

The mean triglyceride was $\mathbf{2 . 4 1} \mathrm{mmol} / \mathrm{L}$

The mean cholesterol was $\mathbf{5 . 1 5}$ mmol/L

The mean fasting insulin was $\mathbf{1 5 . 1 1} \mu \mathrm{U} / \mathrm{m}$

Risk with [lifestyle intervention]

The mean fasting blood glucose (changed

from baseline) was $-\mathbf{0 . 3 2} \mathbf{~ m m o l} / \mathrm{L}$

The mean postprandial Blood Glucose (changed from baseline) was $-\mathbf{0 . 7 7} \mathbf{~ m m o l} / \mathrm{L}$

\section{Risk with [lifestyle intervention]}

The mean fasting blood glucose (changed

from baseline) was $-\mathbf{0 . 8 7} \mathbf{~ m m o l} / \mathrm{L}$

The mean postprandial Blood Glucose (changed from baseline) was $\mathbf{- 1 . 3 4 ~} \mathrm{mmol} / \mathrm{L}$

The mean triglyceride(changed from baseline) was $\mathbf{- 0 . 4 1} \mathrm{mmol} / \mathrm{L}$ MODERATEC

$\oplus \oplus \oplus \bigcirc$ MODERATE
The mean cholesterol(changed from baseline) was $-\mathbf{0 . 4 5} \mathrm{mmol} / \mathrm{L}$
Risk difference with [CHM plus hypoglycemic drugs] MD 0.93 mmol/L lower

(1.1 lower to 0.76 lower)

MD $1.46 \mathrm{mmol} / \mathrm{L}$ lower

(1.73 lower to 1.2 lower)

MD $\mathbf{0 . 8 4 \%}$ lower

(1.05 lower to 0.64 lower)

MD 0.4 mmol/L lower

(0.51 lower to 0.29 lower)

MD 0.4 mmol/L lower

(0.58 lower to 0.22 lower)

MD $1.03 \mu \mathrm{U} / \mathrm{ml}$ lower

(2.35 lower to 0.29 higher)

Risk difference with [CHM] MD $\mathbf{0 . 6 2} \mathbf{~ m m o l} / \mathbf{L}$ lower (1.28 lower to 0.04 higher)

MD $2.14 \mathrm{mmol} / \mathrm{L}$ lower (2.81 lower to 1.47 lower)

Risk difference with [CHM] MD 1.08 mmol/L lower (1.86 lower to 0.30 lower)

MD $1.23 \mathrm{mmol} / \mathrm{L}$ lower (1.96 lower to 0.50 lower)

MD $0.3 \mathbf{~ m m o l} / \mathbf{L}$ lower (0.69 lower to 0.09 higher)

MD 0.66 mmol/L lower

(1.1 lower to 0.23 lower)

Treatment duration 12 weeks

${ }^{*}$ The risk in the intervention group (and its 95\% confidence interval) is based on the assumed risk in the comparison group and the relative effect of the intervention (and its $95 \%$ Cl). CHM, Chinese herbal medicine; Cl, Confidence interval; GRADE, Grading of Recommendations Assessment, Development and Evaluation; MD, Mean difference; RCTs, randomized controlled trials;

Explanations

${ }^{a}$ High statistical heterogeneity, $p<0.05 ;{ }^{b}$ Funnel plot not symmetrical; ' ${ }^{c}$ Small sample size.

Bold values is used to highlight. 
formulae containing Shanyao may improve glycolipid metabolism and fasting insulin level.

\section{Experiment Research Evidence of Shanyao for Diabetes}

The major component groups of Shanyao are saponins, phenolic compounds, sterols, and mucilage (8). Identified active ingredients of Shanyao include polysaccharides, flavonoids, allantoin, choline, dioscin, and so on (9-14). Shanyao has shown immunomodulatory and anti-inflammatory effects (78, 79). Hpyoglycemic effects of Shanyao as food and pharmacological effects in relation to T2DM are reviewed below.

\section{Nutritional Study on the Hypoglycemic Effect of Shanyao}

Shanyao has been studied to explore its effect on blood glucose as a food. A study indicated that meal A (maize flour meal) was composed of $81 \%$ carbohydrate, $3 \%$ protein, and $11 \%$ fat; meal B (cassava flour meal) was composed of $76 \%$ carbohydrate, $3 \%$ protein, and $15 \%$ fat; while meal C (yam flour meal) was composed of $85 \%$ carbohydrate, $2 \%$ protein, and $8 \%$ fat. Analysis of the results demonstrated a better glycemic response with meals A and C compared with meal B; Shanyao as food may bring more benefits to blood glucose (80).

Shanyao's glycemic index has also been investigated. Glycemic index was introduced to rank how slowly or quickly carbohydrate containing foods are digested and increase postprandial blood glucose (24). A study has shown that the actual calorie input may be much lower in Shanyao than in brown rice and white bread, showing that the glycemic value of Shanyao is lower than brown rice (81), which is beneficial for controlling postprandial blood glucose, in turn may benefit diabetic patients.

An experimental research conducted in Brazil showed that Shanyao flour alleviated the consequences of the experimental diabetic disease. It showed that Shanyao flour could control the rise in blood glucose levels in diabetic rats and significantly greater radiodensity of femoral head when compared to DM group, suggesting protection in oxidative agents and postpone bone damage caused by diabetes (82).

Different Shanyao species are present depending on place of production. Dioscorea alata is known to have the highest yields among the Shanyao species with tubers. Dioscorea alata has shown to have higher amylose and total dietary fiber contents, resulting in slower absorption rates and can be particularly useful in diets for diabetics (83).

\section{Pharmacological Effects in Relation to Diabetes of Shanyao and Its Compounds}

The pharmacological effects and preventative effects for T2DM of Shanyao or its compounds have been tested in various animal models.

As we mentioned earlier on, a popular formula used for DM is Liu wei di huang wan made up of six herbs including Shanyao. In fructose-rich chow fed rats, after feeding of Liu wei di huang wan at $26 \mathrm{mg} / \mathrm{kg}$ for $60 \mathrm{~min}$, reduction in plasma glucose was observed
(84). When Shanyao was removed from the mixture, plasma glucose was not modified while this action was not modified by the removal of the other five herbs, indicating important hypoglycemic roles of Shanyao (84). Further, Shanyao produced similar hypoglycemic effects of the Liu wei di huang wan formula, while other herbs in the formula failed to produce the same effects. The authors also investigated the role of Shanyao in improving insulin sensitivity and found that oral administration of Shanyao at $4.2 \mathrm{mg} / \mathrm{kg}$ three times daily into streptozotocin-induced diabetic rats increased the response to exogenous insulin (84).

In vehicle-controlled mice and in alloxan-induced diabetic mice, Shanyao decoction concentrate at $300 \mathrm{mg}$ and $600 \mathrm{mg} / \mathrm{kg}$ for 10 consecutive days can significantly reduce blood glucose level (85). Further, Shanyao has shown preventative effects on induced blood glucose elevation due to different causes including adrenaline, alloxan, and glucose feeding (85). As for hypoglycemic effects, Shan yao can reduce total cholesterol and triglyceride levels in diabetic rats, showing lipid lowering effects (86).

\section{Polysaccharides}

In alloxan-induced diabetic rats and mice, high dose DOTP-80 water-soluble polysaccharide $(400 \mathrm{mg} / \mathrm{kg})$ had strong hypoglycemic activity (87). Moreover, water-soluble polysaccharide could increase the level of antioxidant enzyme (superoxide dismutase) activity in alloxan-induced diabetic mice and stimulated an increase in glucose disposal in diabetic rats (87).

In high fat fed streptozotocin-induced type 2 diabetic rats, Shanyao polysaccharide administration significantly reduced fasting plasma glucose levels, increased serum insulin levels, and decreased glucagon levels, showing hypoglycemic effects $(88,89)$. The hypoglycemic effects of Shanyao polysaccharides are comparable to metformin $(88,89)$.

In vitro testing of Shanyao polysaccharide (YP-1) from a variety of Chinese yam revealed that YP-1 could stimulate ConA-induced $\mathrm{T}$ lymphocyte proliferation, and its branches are extremely important for the expression of the enhancement of the immunological activity (90). Considering the important role of the immune system in the progression of T2DM, YP-1 could play a part in enhancing immunological activities in T2DM.

Shanyao polysaccharides have shown antioxidant activities. Purified Shanyao polysaccharide could scavenge hydroxyl radical and superoxide radical. Additionally, it displayed inhibitory activity against $E$. coli, with a minimal inhibitory concentration of $2.5 \mathrm{mg} / \mathrm{ml}(91)$.

Dexamethasone-induced insulin resistance glucose/lipid metabolism diabetic mice model was established to evaluate the hypoglycemic effect of different concentrations of HuaiShanyao and different molecular weights of polysaccharide HSY-I, HSY-II, and HSY-III. The results indicated that the Chinese yam polysaccharide mixture had hypoglycemic effect (92).

\section{Saponins and Flavones}

The metabolic syndrome is a term for cluster of multiple metabolic risk criteria which is positively correlated with type 
2 diabetes mellitus. Shanyao dioscorin interventions exhibit improved metabolic syndrome activities in obese rats, and peptic hydrolysates of Shanyao dioscorin in vitro exhibit DPP IV inhibitory activities (93).

After intragastric administration of dioscoreae flavone in diabetic mice, it was found that blood glucose of diabetic mice was decreased, the amount of drinking water decreased, and the weight of mice recovered (94). Further experiments showed that dioscoreae flavone could inhibit $\alpha$-glucosidase activity, effectively reduce superoxide dismutase activity and malondialdehyde content in diabetic mice, and have antioxidant effect (94).

Experimental results show that the saponins and flavones of Shanyao had a marked inhibition effect on $\alpha$-amylase. The kinetic analysis indicates that the inhibition type of saponins and flavones on $\alpha$-amylase was competitive, and the enzymesubstrate apparent dissociation constant $\left(\mathrm{Km}^{\prime}\right)$ of acarbose (the control), saponins, and flavones is $118.86,79.23,49.51 \mathrm{mg} / \mathrm{ml}$, respectively (95).

\section{Allantoin}

Allantoin is known as the active principle richly contained in Shanyao (Dioscorea spp.). It is identified as an abundant and active component in Shanyao (Dioscorea spp.) (96). Allantoin may improve glucose utilization in the skeletal muscle through $\beta$-endorphin dependent- and independent-pathways that decrease plasma glucose in STZ-diabetic rats (97).

Allantoin can increase $\beta$-endorphin release through activation of I-2A receptors to lower blood glucose. Also, allantoin can activate I-2B receptors in the skeletal muscle or adipose tissues and brain and others to reduce blood glucose in STZ-diabetic rats. Both actions of allantoin may assist the increase of insulin sensitivity in diabetic rats (98).

\section{Ligands}

$\alpha$-Glucosidase inhibitors are widely used in the treatment of patients with T2DM, which delay the absorption of carbohydrates from the small intestine and result in lowered postprandial blood glucose and insulin levels (99). Two ligands identified as 2,4-dimethoxy-6,7-dihydroxyphenanthrene and batatasin I were extracted from Shanyao using $\alpha$-glucosidase functionalized magnetic nanoparticles as a solid phase extraction absorbent. Their $\alpha$-glucosidase inhibitory activities were significantly higher than acarbose (100).

\section{DISCUSSION}

\section{Summary of Evidence}

In this study, we searched clinical studies and experimental studies to present an overall picture of the evidence for Shanyao and its formulae for additional benefits to conventional therapies in the management of DM. We also explored the possible mechanisms of actions for Shanyao.

This study presents a comprehensive and up-to-date evidence for the treatment of T2DM with Shanyao formulae. A systematic review of 53 randomized controlled trials was identified in English- and Chinese-language databases, and meta-analysis was conducted to evaluate the additive benefits and safety of Shanyao formulae. Systematic evaluation of modern literatures suggested that in terms of controlling blood glucose, blood lipid and improving insulin resistance, traditional Chinese medicine formulae containing Shanyao combined with conventional therapies do show added benefits when compared to hypoglycemic agents and lifestyle intervention alone.

Systematic review of clinical studies indicates that Shanyaocontaining herbal formulae can improve important outcome measures for T2DM including FBG, 2hPG, $\mathrm{HbA}_{1 \mathrm{c}}, \mathrm{TG}, \mathrm{TC}$, and IR in people with T2DM. Further, the adverse events in the herbal formula group are lower than in the control group, suggesting a good safety profile. Taken together, Chinese herbal medicine therapy including Shanyao can be of beneficial for patients with T2DM. The heterogeneity in our meta-analysis was high. We performed a subgroup analysis based on pre-set conditions, but the results of subgroup analysis did not fully explain the source of heterogeneity. Considering different herb usages in different clinical trials, this could be a possible source of heterogeneity in the meta-analysis. Therefore, it is necessary to interpret these results with caution.

The occurrence of DM in the current day and age is closely related to lifestyle change. We found that Shanyao 山药, Fuling 茯苓, Maimendong麦门冬, Shanzhuyu 山茱英, Haungqi 黄芪, Shengdihuang 生地黄, Gegen 葛根, Danshen 丹参, Tianhuafen 天花粉, and Huanglian 黄连are used more in modern literatures. The use of Chinese herbs that taste sweet and bitter, has the effect of invigorating $q i$ and nourishing yin, infiltrating dampness and solidifying astringency, clearing heat, generating body fluid, and detoxifying. In recent years, the prevalence of diabetes in obese and overweight people has doubled in modern China (6). TCM believes that phlegm-dampness is the basic cause of obesity. An ancient practitioner Zhu Danxi clearly put forward in "Dan $X i$ Zhi Fa Xin Yao丹溪治法心要” that “people who are obese have excessive dampness and phlegm in the body". Obesity combined with diabetes is characterized by yin deficiency, internal heat, and phlegm dampness. Therefore, modern diabetes treatment focuses on nourishing yin and clearing heat, drying dampness and resolving phlegm.

To sum up the experimental evidence, Shanyao as a food, is rich in dietary fiber with low glycemic index, which is beneficial to improving postprandial blood glucose of diabetic patients. In diabetic animals, Shanyao can reduce blood sugar, regulate blood lipid, resist oxidation and be beneficial to the bone. The main active ingredients of Shanyao can regulate blood glucose by improving insulin resistance, inhibiting $\alpha$-glucosidase activity, delaying the absorption of glucose in intestine, inhibiting DPPIV activity, increasing the concentration of endogenous GLP-1, antioxidation and regulating immunity.

\section{Limitations of the Current Review}

The included clinical studies also present methodological shortfalls. In randomized clinical trials, appropriate randomization and allocation concealment methods can reduce 
bias, but only $25.81 \%$ of the included studies described the randomization methods, and only $2.58 \%$ of the studies described allocation concealment. Inappropriate randomization and allocation concealment may exaggerate the efficacy. Inadequate blinding method can also lead to overestimation of the effect. Chinese herbal medicine has many obstacles to the implementation of blinding in clinical trials due to the herbal preparation and odor; it is easy for participants to identify which group they are in. Active exploration of the preparation of placebo and the implementation of blinding method of traditional Chinese medicine may be a way to solve this problem. In addition, the included studies did not publish study protocols, and the non-standardization of clinical research reports is not helpful to the dissemination and recognition of research results.

Modern literature systematic reviews included the study of traditional Chinese medicine formulae containing Shanyao. The included studies all used Shanyao as part of a traditional Chinese medicine formula for T2DM. Therefore, the results of modern literature analysis do not fully represent the role of Shanyao in the treatment of T2DM in reducing blood sugar, lipid and improving IR.

\section{Implications in Research and Clinical Practice}

Future clinical studies should be recommended to design and report data following the items required by the Consolidated Standards of Reporting Trials (CONSORT) (101) and its extensions for herbal medicine and traditional CM $(102,103)$.

Rigorous methodology is recommended when designing future clinical trials with correct methods of sequence generation and allocation concealment. Protocols should be published and be registered to minimize reporting bias and increase transparency in the reporting the results.

The included studies reported outcome measures directly related to blood glucose metabolism, lipid profiles, and $\beta$-cell function. As we know, T2DM is a chronic and progressive disease, and those who are affected may have drastic lifestyle changes. Other clinically important outcomes such as quality of life would provide another aspect of understanding the effect of CM therapies for T2DM.

As we all know, T2DM is a progressive and lifelong disease. The included clinical studies had treatments between 2 and 24 weeks with only few studies reporting on follow-up data. Future

\section{REFERENCES}

1. Federation. ID. IDF Diabetes Atlas. 9th edn. Belgium (2019). Available at: https://www.diabetesatlas.org. [cited 2020 March 23rd].

2. Association AD. Classification and Diagnosis of Diabetes: Standards of Medical Care in Diabetes-2020. Diabetes Care (2020) 43:s14-31. doi: $10.2337 / \mathrm{dc} 20-\mathrm{S} 002$

3. Wang L, Gao P, Zhang M, Huang Z, Zhang D, Deng Q, et al. Prevalence and Ethnic Pattern of Diabetes and Prediabetes in China in 2013. Jama (2017) 317:2515-23. doi: 10.1001/jama.2017.7596 studies may consider using longer treatment durations and lengthy follow-up period to reflect clinical practice and provide evidence for the long-term effects of CM treatments using Shanyao.

Although we present in the study available experimental evidence for Shanyao, the volume of studies is small compared to other commonly used herbs such as ginseng. More experimental study data will improve the understanding of mechanisms of action of hypoglycemic effects of Shanyao. Results may provide future directions for the development of new and improved hypoglycemic agents.

Based on clinical studies, herbs that are commonly used with Shanyao in the modern context include huangqi 黄華, shengdihuang 生地黄, gegen 葛根, tianhuafen 天花粉, danshen 丹参, fuling 获苓, maimendong 麦门冬, shanzhuyu 山茱莫, and huanglian 黄连.

Finally, this study provides comprehensive information about Shanyao's use for T2DM in clinical studies. At the same time, it explores possible mechanisms through experimental studies, providing evidence of hypoglycemic mechanism of Shanyao at a cellular and animal model levels.

\section{DATA AVAILABILITY STATEMENT}

The raw data supporting the conclusions of this article will be made available by the authors, without undue reservation.

\section{AUTHOR CONTRIBUTIONS}

All authors listed have made a substantial, direct, and intellectual contribution to the work and approved it for publication.

\section{FUNDING}

The China-Australia International Research Centre for Chinese Medicine (CAIRCCM) - a joint initiative of RMIT University, Australia and the Guangdong Provincial Academy of Chinese Medical Sciences, China supported this research. We also received funding from the Ministry of Science \& Technology of China (grant number 2015BAI04B00) and Guangdong Provincial Hospital of Traditional Chinese Medicine (grant numbers 2018DB03). 
7. Shi Y. Internal medicine of traditional Chinese medicine (Textbook for the 13th five year plan of general higher education, textbook for the plan of national higher medical institutions). Beijing, China: Science Press (2017).

8. Bensky D, Clavey S, Stoger E. Chinese herbal medicine Materia Medica. 3rd ed. Seattle, US: Eastland Press, Inc (2004).

9. Yang MH, Chin YW, Yoon KD, Kim J. Phenolic compounds with pancreatic lipase inhibitory activity from Korean yam (Dioscorea opposita). J Enzyme Inhibition Med Chem (2014) 29:1-6. doi: 10.3109/14756366.2012.742517

10. Yang MH, Yoon KD, Chin YW, Park JH, Kim J. Phenolic compounds with radical scavenging and cyclooxygenase-2 (COX-2) inhibitory activities from Dioscorea opposita. Bioorg Med Chem (2009) 17:2689-94. doi: 10.1016/ j.bmc.2009.02.057

11. Kong XF, Zhang YZ, Yin YL, Wu GY, Zhou HJ, Tan ZL, et al. Chinese yam polysaccharide enhances growth performance and cellular immune response in weanling rats. J Sci Food Agricult (2009) 89:2039-44. doi: 10.1002/ jsfa. 3688

12. Jeon JR, Lee JS, Lee CH, Kim JY, Kim SD, Nam DH. Effect of Ethanol Extract of Dried Chinese Yam (Dioscorea batatas) Flour Containing Dioscin on Gastrointestinal Function in Rat Model. Arch Pharm Res (2006) 29:348-53. doi: $10.1007 / \mathrm{bf} 02968583$

13. Fu YC, Ferng LH, Huang PY. Quantitative analysis of allantoin and allantoic acid in yam tuber, mucilage, skin and bulbil of the Dioscorea species. Food Chem (2006) 94:541-9. doi: 10.1016/j.foodchem.2004.12.006

14. Fu YC, Chen SH, Huang PY, Li YJ. Application of Bubble Separation for Quantitative Analysis of Choline in Dioscorea (Yam) Tubers. J Agric Food Chem (2005) 53:2392-6. doi: 10.1021/jf048501h

15. Green J. Cochrane Handbook for Systematic Reviews of Interventions(Version 5.1.0). UK: The Cochrane Collaboration (2011). March.

16. World Health Organisation. Definition, Diagnosis and Classification of Diabetes Mellitus and its Complications, Part 1: Diagnosis and Classification of Diabetes Mellitus. Geneva (1999).

17. Chinese Diabetes Society. Prevention and Treatment of Type 2 Diabetes Mellitus in China (2010 version). Beijing China: Beijing University Publisher (2011).

18. Chinese Diabetes Society. Prevention and Treatment of Type 2 Diabetes Mellitus in China (2013 version). Chin J Diabetes (2014) 6:447-98. doi: 10.3760/cma.j.issn.1000-6699.2014.10.020

19. Zhou ZY. Internal medicine of traditional Chinese medicine / national planning textbook for "12th Five Year Plan", "11th Five Year Plan" and "15th five year plan" of Higher Education. Beijing, China: China Press of Traditional Chinese Medicine (2007).

20. Zhang BY. Internal medicine of traditional Chinese medicine / teaching materials of medical colleges and Universities. Shanghai, Chian: Shanghai Science and Technology Press (1985).

21. Zhang BL. Internal medicine of traditional Chinese medicine / textbook for the 13th five year plan of higher education in the industry of traditional Chinese Medicine. Beijing, China: China Press of Traditional Chinese Medicine (2017).

22. Fan GJ. Series on clinical diagnosis and treatment of specialist diseases in traditional Chinese Medicine - clinical diagnosis and treatment of specialist diseases in endocrinology and rheumatism in traditional Chinese medicine. 2nd Edition. Beijing, China: People's Medical Publishing House (2013).

23. Wu MH. Internal medicine of traditional Chinese medicine / textbook for the 12th Five Year Plan of higher education in the industry of traditional Chinese Medicine. Beijing, China: China Press of Traditional Chinese Medicine (2012).

24. Wolever TM. The glycemic index. World Rev Nutr Diet (1990) 62:120-85. doi: 10.1007/s11883-007-0064-x

25. Li WT. The effect of Dioscorea porridge on blood sugar and blood lipid in patients with type 2 diabetes mellitus. Master dissertation. Fuzhou: Fujian University of Traditional Chinese Medicine (2016).

26. Zhang LY. Clinical Observation on the Treatment of Damp-heat Syndrome in Type 2 Diabetes Mellitus by Clearing Heat and Removing Dampness. Master dissertation. Beijing: Beijing University of Chinese Medicine (2016).

27. Wang GM, Wang ZG. Clinical Study on the Treatment of Insulin Resistance in Type 2 Diabetes Mellitus with the Method of Benefiting Kidney and Strengthening Spleen [Article in Chinese]. Gansu J TCM (2009) 22:25-6. doi: $10.3969 /$ j.issn.1004-6852.2009.04.016
28. Wu Z. Study on Jianpi Jiangtang Decoction in the Treatment of Spleen Deficiency and Phlegm Stasis Syndrome of Diabetes Mellitus. Master dissertation. Nanjing: Nanjing University of Traditional Chinese Medicine (2005).

29. Liu Y. Huang Qi Tang in type 2 diabetes Qi and Yin Deficiency and Blood Stasis Syndrome Clinical Study. Master dissertation. Xianyang: ShanXi University Of Chinese Medicine (2010).

30. Chen Y. The Clinic research of treating Type 2 Diabetes by thetherapy of nourishing spleen and kidneys,heat-clearing and yin -nourishing. Master dissertation. Nanjing: Nanjing University of Traditional Chinese Medicine (2012).

31. Zhang XX. Clinical Observation and Mechanism Study on the Effect of Shenqi Compound and Insulin on Cell Function, Oxidative Stress and Blood Level in Patients with Diabetes Mellitus. Master dissertation. Chengdu: Chengdu University of Chinese Medicine (2013).

32. Li XL. Clinical studies on insulin from the intestine to explore the mechanism of Spleen Qi Treatment of type 2 diabetes. Master dissertation. Jinan: Shandong University Of Chinese Medicine (2014).

33. Wang SL. The early Protective effect on $\beta$-cell function in Type 2 diabetes with the therapy of nourishing yin and clearing away heat. Master dissertation. Guangzhou: Guangzhou University of Chinese Medicine (2010).

34. Zhang N. Clinical Study on Invigorating Qi and Nourishing Yin Therapy for Insulin Resistance in Initial Type 2 Diabetes Mellitus. Master dissertation. Taian: Tianshan Medical University (2013).

35. Lv P, Chen L, Bi NN. Clinical Study on Yuye Decoction in Treatment of Type 2 Diabetes with Qi and Yin Deficiency Syndrome [Article in Chinese]. China J Chin Med (2018) 33:1244-7. doi: 10.16368/j.issn.1674-8999.2018.07.294

36. Peng YZ, Xu Y. Clinical efficacy of the Shenmai Jiangtang tablet on senile patients with type 2 diabetes mellitus of Qiyin Liangxu [Article in Chinese]. Clin Res Tradit Chin Med (2018) 10:67-9. doi: CNKI:SUN:ZYLY.0.2018-06-031

37. Wang ML. Clinical Observation of tang yi prescription on Improving Symptom of Diabetes mellitus Patients. Master dissertation. Guangzhou: Guangzhou University of Chinese Medicine (2012). p. 15-24.

38. Zhang LL. Clinical Observation of Yiqi Yangyin Jiangtang Decoction in the Treatment of Type 2 Diabetes Mellitus. Master dissertation. Beijing: Beijing University of Chinese Medicine (2015).

39. Yang LF. Clinical Study of Qianjin Wenwu Decoction Combined with Metformin in the Treatment of Type 2 Diabetes in Taiyin People. Master dissertation. Yanji: Yanbian University (2017).

40. Lin JC. Observation on the curative effect of traditional Chinese medicine combined with Baduanjin on type 2 diabetes mellitus. Master dissertation. Nanjing: Nanjing University of Traditional Chinese Medicine (2011).

41. Zhang J. Clinical Observation and Gastrointestinal Hormone Study on Diabetic Spleen Deficiency Syndrome Treated by Jianpi Yiqi Method. Master dissertation. Jinan: Shangdong University of Chinese Medicine (2012).

42. Bai HM. 40 Cases of Type 2 Diabetes Treated with Yiqi Yangyin Huoxue Decoction [Article in Chinese]. Shanxi Tradit Chin Med (2006) 27:1237328. doi: 10.3969/j.issn.1000-7369.2006.10.053

43. Wang HQ. The Clinical Research of combination of sweet and bitter herbs for the yin dificiency and heat accumulation of Type 2 diabetes millitus. Master dissertation. Jinan: Shandong University Of Chinese Medicine (2016).

44. Zhang XX, Liu Y, Xiong DQ, Xie CG. Insulin combined with Chinese medicine improves glycemic outcome through multiple pathways in patients with type 2 diabetes mellitus. J Diabetes Investigation (2015) 6:708-15. doi: $10.1111 /$ jdi.12352

45. Zhu LB, Li J. Effect of Jiangtangjing Granule on Prethrombotic State in Elderly Patients with Type 2 Diabetes Mellitus [Article in Chinese]. J Gansu Coll Tradit Chin Med (2013) 30:31-4. doi: CNKI:SUN:GSZX.0.2013-05-016

46. Zhu LY, Wu ZW, Cheng ZJ. Clinical Curative Effect of self-made Herbal Medicine combined with western medicine on diabetes [Article in Chinese]. Liaoning J Tradit Chin Med (2015) 42:561-3. doi: 10.13192/j.issn.10001719.2015.03.048

47. Zhou YH, Zhong ZY, Deng LH, Pen NX, Shen QS. Effect of Erban Decoction on the Blood Sugar of Senile patients with type 2 diabetes [Article in Chinese]. J New Chin Med (2014) 46:90. doi: 10.13457/j.cnki.jncm.2014.10.037

48. Zhou JY, Shen W. Clinical Observation of Shenqi Ningxiao Decoction in the Treatment of Type 2 Diabetes Mellitus [Article in Chinese]. JClin Chin Med (2009) 21:204-5. doi: 10.16448/j.cjtcm.2009.03.007 
49. Zhou JJ, Dong YY. Clinical Study of Yiqi Yangyin Capsule in the Treatment of Diabetes Mellitus (Type 2 Diabetes Mellitus) with Deficiency of Qi and Yin [Article in Chinese]. Drugs Clin (2012) 8:252-3.

50. Zhou DQ, Feng P, Wan P. Clinical Observation on 50 Cases of Type 2 Diabetes Treated with Jiangtang Mixture [Article in Chinese]. Zhejiang J Tradit Chin Med (2008) 43:431. doi: 10.3969/j.issn.0411-8421.2008.07.047

51. Zhao FJ, Song J. Clinical Study on the Treatment of Type 2 Diabetes Mellitus with the Method of Supplementing Qi and Nourishing Yin [Article in Chinese]. J Heze Med College (2009) 21:39-41. doi: 10.3969/j.issn.10084118.2009.01.019

52. Zhang FB, Tao Y, Ning J. Observation on the efficacy of no-named Chinese herbal Formula in the adjuvant treatment of type 2 diabetes mellitus [Article in Chinese]. Chin Tradit Med Sci Technol (2015) 22:186-7. doi: CNKI:SUN: TJYY.0.2015-02-034

53. Zhang YL, Zhang J, Xie FM. Combined Chinese and Western Medicine for 98 Cases of Secondary Type 2 Diabetes Mellitus Irresponsive Sulfonylurea Drugs [Article in Chinese]. Shanghai J Tradit Chin Med (2004) 38:18-9. doi: CNKI:SUN:SHZZ.0.2004-07-007

54. Zhang HX, Chang FY, Zhang BR, Deng GX, Yu YX, Zhang GL. Clinical Observation on 109 Cases of Insulin Resistance in Type 2 Diabetes Mellitus Treated with Xiaotangping Capsule [Article in Chinese]. J Tradit Chin Med (2007) 48:812-3. doi: 10.3321/j.issn:1001-1668.2007.09.016

55. Zhang WJ, Yang HJ, Zhang ZJ, Zhang ZX, Wu LL, Chen M. Effect of Qilian Xiaoke Formula on Diabetes Insulin Resistance and Related Inflammatory Factors [Article in Chinese]. Hunan J Tradit Chin Med (2011) 27:4-6. doi: 10.3969/j.issn.1003-7705.2011.01.002

56. Zhang HH, Pan BY. Clinical Observation on 120 Cases of Type 2 Diabetes Mellitus with Deficiency of both Qi and Yin and Blood Stasis Treated by Combination of Traditional Chinese Medicine and Western Medicine [Article in Chinese]. Beijing J Tradit Chin Med (2009) 28:538-40. doi: 10.3969/j.issn.1002-2619.2009.06.011

57. Zhang GQ, You Q, Lv M, Chen DL. 36 Cases of Type 2 Diabetes Treated with Yiqi Yangyin Huoxue Decoction [Article in Chinese]. New World Diabetes (2014) 11:35-6. doi: CNKI:SUN:TNBX.0.2014-22-031

58. Zhang GZ, Sun QF, Guo JF, Huang BT, Li SH, Wang FH, et al. Clinical study on the treatment of type 2 diabetes mellitus with integrated traditional Chinese and Western Medicine [Article in Chinese]. Modern J Integrated Tradit Chin Western Med (2002) 11:5-7. doi: 10.3969/j.issn.10088849.2002 .01 .003

59. Zhang B, Nan Z, Xu CM, Liu GP, Guo XT, Yang LY. et al. Clinical Observation on 30 Cases of Type 2 Diabetes Treated by Combination of Traditional Chinese Medicine and Western Medicine [Article in Chinese]. Hunan J Tradit Chin Med (2017) 33:13-5. doi: 10.16808/j.cnki.issn10037705.2017.08.005

60. Yu CB, Yan DB, Zhang MZ, Chen Y, Wang Y. Clinical Observation of Selfmade Taiping Tangke Concentrated Pill in the Treatment of Type 2 Diabetes Mellitus [Article in Chinese]. Pract Clin Appl Integrated Tradit Chin Western Med (2013) 13:16-7. doi: 10.3969/j.issn.1671-4040.2013.01.010

61. Yu ZL, Zhou ZK, Quan FL. Clinical Observation on 30 Cases of Type 2 Diabetes Treated by Combination of Traditional Chinese Medicine and Western Medicine [Article in Chinese]. Modern J Integrated Tradit Chin Western Med (2004) 13:186. doi: 10.3969/j.issn.1008-8849.2004.02.037

62. Xu XJ, Liu D, Zhang W, Wang J. Study of the effect of add itive Shenling Baizhu san decoction to insulin sensitivity index of patients with type 2 Diabetes Mellitus [Article in Chinese]. Clin J Tradit Chin Med (2015) 27:954-6. doi: $10.16448 /$ j.cjtcm.2015.0360

63. Xie MH, Lu CM. Observation on the therapeutic effect of Dihuang Jiangtang Pill on type 2 diabetes mellitus [Article in Chinese]. Jilin J Tradit Chin Med (2007) 27:22-3. doi: 10.13463/j.cnki.jlzyy.2007.06.016

64. Tang Y, Li JS. Research on Treating Syndrome of Endogenous Heat Due to Yin Deficiency Type 2 Diabetes Patients with Liuwei Dihuang Bolus Combined Metformin Hydrochloride Tablets [Article in Chinese]. J Huan Normal Univ (Med Sci) (2015) 12:28-31. doi: CNKI:SUN:HNYG.0.2015-04010

65. Shang J, Zheng JQ, Su XL. Effect of integrated traditional Chinese and Western Medicine on insulin resistance and islet beta cell function in type 2 diabetes mellitus [Article in Chinese]. Guangming J Tradit Chin Med (2014) 29:1197-200. doi: 10.3969/j.issn.1003-8914.2014.06.038
66. Peng MN, Wang HZ, Zheng CF. Observation of the Effect of Using Ginseng and Astragalus Arbran Decoction with Western Medicine on the Type-2 Diabetes [Article in Chinese]. Int J Tradit Chin Med (2009) 31:241. doi: 10.3760/cma.j.issn.1673-4246.2009.03.028

67. Lv Y, Hou JJ, Lu YE. 45 Cases of Type 2 Diabetes Treated by the Method of Supplementing Qi, Nourishing Yin and Promoting Blood Circulation [Article in Chinese]. Shanxi Tradit Chin Med (2011) 32:1485-6. doi: 10.3969/j.issn.1000-7369.2011.11.030

68. Luo XL, Zhao C. Clinical Observation on the Treatment of 52 Cases of DM II With ZhiboDihuang Decoction and Metform [Article in Chinese]. Guiding J TCM (2005) 11:15-7. doi: 10.3969/j.issn.1672-951X.2005.06.008

69. Liu L, Liu DL, Liu HL, Zhao HX, Qu X, Li ZY, et al. On Treatment of 30 Cases of Type 2 Diabetes with Deficiency of Qi and Yin Deficiency Syndrome by Huoxue Jiangtang Potion [Article in Chinese]. Modern Tradit Chin Med (2017) 37:16-20. doi: CNKI:SUN:XDZY.0.2017-05-007

70. Liu C, Ge H, Li M, Li SY, Yuan W, Zhang J. Effect of Yiqi Yangyin Qingre Formula on serum levels of blood lipids, blood gluose and 1,2 5dihydroxyvitamin D3 in patients with type 2 diabetes mellitus [Article in Chinese]. Chin J Biochem Drugs (2016) 36:145-7. doi: CNKI:SUN: SHYW.0.2016-02-050

71. Li ZH, Yang M, Tang D. Shen Li. Clinical Observation of Jiangtang Capsule Combined with Western Medicine in Treating 102 Cases of Type 2 Diabetes with Deficiency of Qi and Yin [Article in Chinese]. Guiding J TCM Pharmacy (2011) 17:34-6. doi: 10.3969/j.issn.1672-951X.2011.02.016

72. Hou YH. Clinical Study of Treating Type 2 Diabetes Mellitus with the Method of Smoothing the Liver and Strengthening The Spleen. Master dissertation. Jinan: Shandong University of Traditional Chinese Medicine (2011).

73. Gao L, Chen YY, Huo GS, Wan YX. Clinical efficacy of metformin combined with Shenqi Jiangtang Capsule in the treatment of type 2 diabetes mellitus and its influence on blood sugar, lipid and inflammatory factors [Article in Chinese]. Chin J Clin Rational Drug Use (2017) 10:1-3. doi: 10.15887/ j.cnki.13-1389/r.2017.36.001

74. Fan YL, Huang XJ, Gao JN, Chen HW. Clinical Observation of Shenqi Jiangtang Granule in the Assistant Treatment of Type 2 Diabetes Mellitus [Article in Chinese]. Chin J Med Equipment (2014) 11:142. doi: CNKI:SUN: YXZB.0.2014-S1-170

75. Cui JH, Luo HX. Observation on curative effect of 40 cases of type 2 diabetes mellitus treated by integrated traditional Chinese and Western Medicine [Article in Chinese]. Yunnan J Tradit Chin Med (2006) 27:4-5. doi: 10.3969/ j.issn.1007-2349.2006.04.003

76. Cao Y, Zou BY. Clinical Observation on Treating 54 Cases of Type 2 Diabetes with Modified Baihurensheng Decoction and Western Medicine [Article in Chinese]. Guiding J TCM (2007) 13:18-20. doi: 10.3969/ j.issn.1672-951X.2007.11.009

77. Cao AM, Zhang GJ, Zheng J, Dan YL. Clinical Observation on 68 Cases of Type 2 Diabetes Treated with Wuhuang Tongmai Decoction [Article in Chinese]. Beijing J Tradit Chin Med (2008) 27:957-8. doi: 10.16025/j.16741307.2008.12.006

78. Kim S, Shin S, Hyun B, Kong H, Han S, Lee A, et al. Immunomodulatory Effects of Dioscoreae Rhizome Against Inflammation through Suppressed Production of Cytokines Via Inhibition of the NF-kappaB Pathway. Immune Netw (2012) 12:181-8. doi: 10.4110/in.2012.12.5.181

79. Lee SC, Tsai CC, Chen JC, Lin CC, Hu ML, Lu S. The evaluation of reno- and hepatoprotective effects of huai-shan-yao (Rhizome Dioscoreae). Am J Chin Med (2002) 30:609-16. doi: 10.1142/S0192415X02000624

80. Bakare-Odunola MT, Mustapha A, Abdu Aguye I. Effect of Nigerian meals on the Pharmacokinetics of chlorpropamide in type II diabetic patients. Eur J Drug Metab Pharmacokinet (2008) 33:31-5. doi: 10.1007/BF03191016

81. Lin MH, Wu MC, Lu S, Lin J. Glycemic index, glycemic load and insulinemic index of Chinese starchy foods. World J Gastroenterol (2010) 16:4973-9. doi: 10.3748/wjg.v16.i39.4973

82. Rego Tde S, Ash Lda S, Pessoa L, Feijo MB, Leite J, dos Santos Ade S, et al. The intake of yam (Dioscorea bulbifera Linn) attenuated the hyperglycemia and the bone fragility in female diabetic rats. Nutricion Hospitalaria (2014) 29:370-5. doi: 10.3305/nh.2014.29.2.7046

83. Faustina Dufie WM, Oduro I, Ellis WO, Asiedu R, Maziya-Dixon B. Potential health benefits of water yam (Dioscorea alata). Food Funct (2013) 4:1496-501. doi: 10.1039/c3fo60064e 
84. Hsu JH, Wu YC, Liu IM, Cheng JT. Dioscorea as the principal herb of DieHuang-Wan, a widely used herbal mixture in China, for improvement of insulin resistance in fructose-rich chow-fed rats. J Ethnopharmacol (2007) 112:577-84. doi: 10.1016/j.jep.2007.05.013

85. Hao ZQ, Hang BQ, Wang Y. Effect of Shan Yao decoction on reducing blood glucose levels in diabetic mice [Article in Chinese]. J China Pharm Univ (1991) 22:158-60. doi: CNKI:SUN:ZGYD.0.1991-03-014

86. Hang YY. Pharmacological experiments on lowing the blood sugar and blood lipids level of Rhizoma Dioscoreae [Article in Chinese]. J Plant Resour Environment (1994) 3:59-60. doi: CNKI:SUN:ZWZY.0.1994-04-012

87. Fan YJ, He QY, Luo AS, Wang MY, Luo AX. Characterization and antihyperglycemic activity of a polysaccharide from Dioscorea opposita Thunb roots. Int J Mol Sci (2015) 16:6391-401. doi: 10.3390/ijms16036391

88. Lv J, Wei PF, Bai F. Effect of Chinese yam polysaccharide on platelet and enzyme activity in type 2 diabetic rats [Article in Chinese]. Chin J Gerontol (2017) 37:3186-7. doi: 10.3969/j.issn.1005-9202.2017.13.027

89. Yang HL, Zhang HX, Li LH, Wang Y, Zhang WW, Jiang X. Study of the hypoglycemic mechanism of Chinese yam polysaccharide in type 2 diabetic rats [Article in Chinese]. J Agricult Univ Hebei (2010) 33:100-3. doi: 10.3969/j.issn.1000-1573.2010.03.022

90. Zhao GH, Kan JQ, Li ZX, Chen ZD. Structural features and immunological activity of a polysaccharide from Dioscorea opposita Thunb roots. Carbohydr Polym (2005) 61:125-31. doi: 10.1016/j.carbpol.2005.04.020

91. Yang WF, Wang Y, Li XP, Yu P. Purification and structural characterization of Chinese yam polysaccharide and its activities. Carbohydr Polym (2015) 117:1021-7. doi: 10.1016/j.carbpol.2014.09.082

92. Li Q, Li WZ, Gao QY, Zou YX. Hypoglycemic Effect of Chinese Yam (Dioscorea opposita rhizoma) Polysaccharide in Different Structure and Molecular Weight. J Food Sci (2017) 82:2487-94. doi: 10.1111/17503841.13919

93. Shih SL, Lin YS, Lin SY, Hou WC. Effects of yam dioscorin interventions on improvements of the metabolic syndrome in high-fat diet-induced obese rats. Bot Stud (2015) 56:4. doi: 10.1186/s40529-015-0084-8

94. He F. Extraction Active components in dioscoreae rhizome and Research on hypoglycemic bioactive. Master dissertation. Chongqing: Southwestern University (2011).

95. Qu S, Zhang LM, Zhang XB, Li ZJ. Inhibition of $\alpha$-Amylase Activities by Extracts of Chinese Yam. In: Proceedings of the 2012 International Conference on Applied Biotechnology (ICAB 2012) SpringerLink (2014). p. 1499-506. doi: 10.1007/978-3-642-37925-3_159

96. Kazahiko Sagara MO. Kejichi Suto, and Tsuguchika Yoshida. Quantitative Determination of Allantoin in Dioscorea Rhizome and an
Oriental Pharmaceutical Preparation, Hachimi-Gan, by HighPerformance Liquid Chromatography. Planta Med (1989) 55:93. doi: 10.1055/s-2006-961841

97. Niu CS, Chen W, Wu HT, Cheng KC, Wen YJ, Lin KC, et al. Decrease of plasma glucose by allantoin, an active principle of yam (Dioscorea spp.), in streptozotocin-induced diabetic rats. J Agric Food Chem (2010) 58:12031-5. doi: $10.1021 /$ jf103234d

98. Lin KC, Yeh LR, Chen LJ, Wen YJ, Cheng KC, Cheng JT. Plasma Glucoselowering Action of Allantoin is Induced by Activation of Imidazoline I-2 Receptors in Streptozotocin-induced Diabetic Rats. Horm Metab Res (2012) 44:14-46. doi: 10.1055/s-0031-1295439

99. van de Laar FA, Lucassen PL, Akkermans RP, van de Lisdonk EH, Rutten GE, van Weel C. $\alpha$-Glucosidase Inhibitors for Patients With Type 2 Diabetes. Diabetes Care (2005) 28:154. doi: 10.2337/diacare.28.7.1840

100. Zhang SS, Wu DD, Li H, Zhu JH, Hu WP, Lu MH, et al. Rapid identification of alpha-glucosidase inhibitors from Dioscorea opposita Thunb peel extract by enzyme functionalized $\mathrm{Fe}_{3} \mathrm{O}_{4}$ magnetic nanoparticles coupled with HPLC-MS/MS. Food Funct (2017) 8:321927. doi: 10.1039/C7FO00928C

101. Schulz KF, Altman DG, Moher D. CONSORT 2010 Statement: updated guidelines for reporting parallel group randomised trials. Trials (2010) 11:32. doi: 10.1016/j.ijsu.2011.09.004

102. Bian Z, Liu B, Moher D, Wu T, Li Y, Shang H, et al. Consolidated standards of reporting trials (CONSORT) for traditional Chinese medicine: current situation and future development. Front Med (2011) 5:171-7. doi: 10.1007/ s11684-011-0132-Z

103. Gagnier JJ, Boon H, Rochon P, Moher D, Barnes J, Bombardier C. Reporting randomized, controlled trials of herbal interventions: an elaborated CONSORT statement. Ann Intern Med (2006) 144:364-7. doi: 10.7326/ 0003-4819-144-5-200603070-00013

Conflict of Interest: The authors declare that the research was conducted in the absence of any commercial or financial relationships that could be construed as a potential conflict of interest.

Copyright (c) 2020 Sun, Di, Lu, Guo, Tang, Zhang, Xue and Fan. This is an openaccess article distributed under the terms of the Creative Commons Attribution License (CC BY). The use, distribution or reproduction in other forums is permitted, provided the original author(s) and the copyright owner(s) are credited and that the original publication in this journal is cited, in accordance with accepted academic practice. No use, distribution or reproduction is permitted which does not comply with these terms. 